\title{
Computer-Assisted Navigation Surgery in Oral and Maxillofacial Surgery
}

\author{
Shintaro Sukegawa and Takahiro Kanno
}

\subsection{Introduction}

The anatomy of the oral and facial region is complex, having many vital organs and structures, including an intricate network of blood vessels and nerves as well as the brain, eyes, nose, and vital teeth. Plain radiography can be used for screening because of its simplicity, but it cannot characterize detailed anatomical relationships. Currently, imaging diagnosis must be performed with computed tomography (CT) and/or magnetic resonance imaging (MRI) before surgery to characterize the surgical area and surrounding anatomical structures. Recent developments in imaging technology have allowed for rapid processing and visualization of significant amounts of data yielded from a variety of digital imaging modalities. Prerequisites have been established for threedimensional (3D) visualization as well as programs for the computer-assisted 3D planning of surgical procedures, and these image sources are now available to assist the surgeon in the operating room [1].

Today, surgeons can use computer-aided design and computer-aided modeling (CAD/CAM) software to assist with the planning and implementation of complex maxillofacial surgical procedures [2]. CAD/CAM software allows the clinician to import two-dimensional (2D) CT data in DICOM format (Digital Imaging and Communications in Medicine) to a computer workstation and create accurate $3 \mathrm{D}$ representations of the facial skeleton and related soft tissues. The data can then be used either to print a stereolithographic (STL) model or for virtual manipulation of the generated 3D model by segmentation, reflection (mirroring), insertion, or repositioning of 3D objects for treatment planning [3].

\section{S. Sukegawa}

Division of Oral and Maxillofacial Surgery, Kagawa Prefectural Central Hospital, Takamatsu, Kagawa, Japan

T. Kanno $(\bowtie)$

Department of Oral and Maxillofacial Surgery, Shimane University

Faculty of Medicine, Izumo, Shimane, Japan

e-mail: tkanno@med.shimane-u.ac.jp
Stereolithographic 3D models are useful for maxillomandibular reconstruction indicators such as

1. A guide for plate adaptation.

2. Planning bony osteotomies.

3. Planning for graft or flap placements.

4. Surgeries involving jaw repositioning.

5. Construction of patient-specific custom implants (PSI).

Not only are they useful in visualizing and planning, but also for providing haptic support to the surgeon for perceived excellent tactile feedback during virtual surgery. They also prove to be very useful in creating a more predictable workflow for the intended surgical intervention. Unfortunately, there is no single prediction method where the surgical plan as performed on the model can be directly transferred to the patient.

Computer-assisted surgery (CAS) uses data from image processing, and can be divided into two major categories:

1. Computer-assisted presurgical planning.

2. Intraoperative navigation.

Computer-assisted presurgical planning involves preoperative surgical simulation using physical or virtual $3 \mathrm{D}$ images or models, and helps the surgeon to appreciate the underlying skeletal anatomy in a more precise manner. The application of preoperative surgical simulations is being used in the field of dental implantology for determining the appropriate positions and sizes of implants as well as evalu- 
ating and planning for bone augmentation when needed, and in the field orthognathic surgery for assessing the amount and direction of movement of the jaws.

The process of intraoperative navigation was developed to improve the sequence of "diagnosis-surgical planningsurgery," allowing surgeons to accurately visualize the positions of surgical instruments and guides in real time on a display of patient CT and/or MRI data. Intraoperative navigation systems integrate diagnostic imaging with the actual surgical field, allowing simultaneous visualization of the surgical site and the analogous image counterpart with the help of a sensor that enables more precise access and manipulation for areas with sensitive anatomy. These navigation systems have now evolved greatly to minimize invasiveness while improving accuracy. The development of intraoperative navigation surgery has enabled improvement in execution and predictability for greater precision during oral-maxillofacial surgery.

This section presents an overview of currently available navigation systems and their applications, focusing on clinical utility and solutions they offer for problems and challenges in the field of oral and maxillofacial surgery.

\subsection{Medical Navigation Technology}

"Navigation-assisted surgery" is a broad term and can be interpreted in various ways.
This is most usefully represented by three questions: "Where is the anatomical target in our patient?", "How can we reach our target safely for our patient?", and "What is our current anatomical location?".

Besides these questions, navigation-assisted surgery may also be used as an "information center" to provide surgeons with accurate and efficiently retrievable information. Navigation systems used for surgery are very similar to a global positioning system (GPS), as is commonly found in automobiles.

It comprises three principal components: (1) a localizer, which is analogous to a satellite; (2) a "hand-held" probe, which corresponds to the track waves emitted by the GPS unit; and (3) the CT scan data of the patient, which is analogous to a road map.

Intraoperative navigation systems were initially developed for use in neurosurgery, but they are now commonly used in surgery of the craniomaxillofacial region due to their high accuracy and reliability $[1,4]$ (Fig. 41.1).
Fig. 41.1 Components of a surgical navigation system. A surgical navigation system is comparable to a global positioning system (GPS) as is commonly used in automobiles, and is composed of three primary components: a localizer, which is analogous to a satellite in space; an instrument or surgical probe, which represents the track waves emitted by the GPS unit in the vehicle; and a CT scan data set, which is analogous to a road map
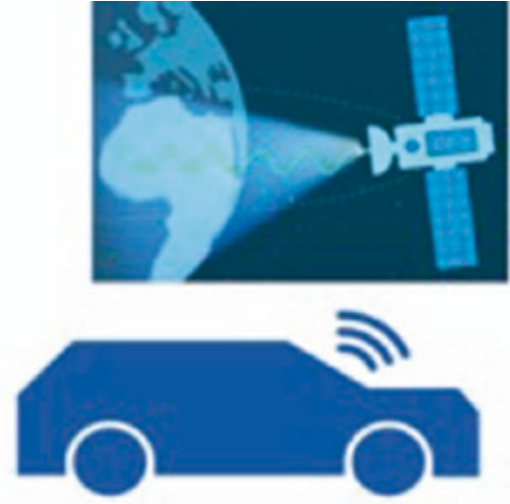

Instrument
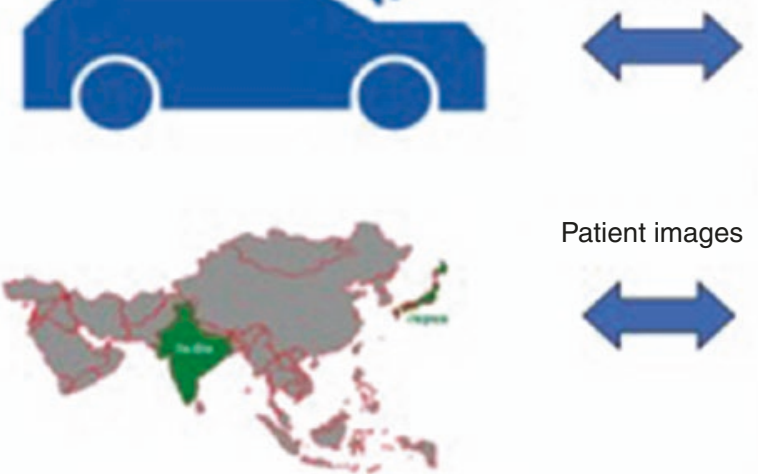

Patient images
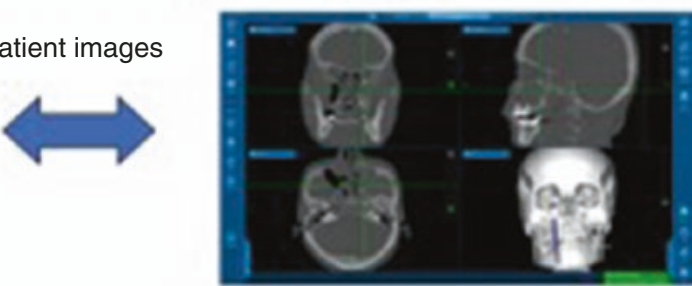

CAssociation of Oral and Maxillofacial Surgeons of India 


\subsubsection{Differences Between Optical and Electromagnetic Tracking Systems}

Two main technologies are currently available for intraoperative navigation, including optical and electromagnetic (Fig. 41.2), and they share the same function. However, they use very different technologies to relay position information to the surgeon. Two major components of the optical navigation system are measured using an infrared camera. These include the position of the reference frame, which is the optical point of reference for navigation and is also called the patient tracker, and a surgical probe with light reflectors. This enables the position of the surgical probe to be displayed on the CT or MRI image in real time. Care should be taken to ensure that both the tracker and the probe are detected by the infrared camera of the navigation system to track the instrument position within the surgical field [5]. By contrast, the electromagnetic system utilizes electromagnetic fields and reference points on a patient borne device, along with a wired instrument for the surgeon to manipulate within the surgical field. The system functions by creating a magnetic field of known intensity and then using microsensors in key instruments to allow the system to determine where the instrument is located relative to the patient's anatomy [6]. In contrast to an optical system, an electromagnetic system does not require a clear line of vision between the IR camera and the sensors, allowing equipment and objects to be placed between the sensors and the IR camera. However, the accuracy of this system is compromised when using ferromagnetic instruments.

\subsubsection{Registration Techniques}

Registration is the task of obtaining the mutual transformation matrix by calculating the relationship between the coordinates of the actual patient space and the coordinates of the medical image. In other words, this procedure involves the alignment of the patient and the image, and it is the most important process when performing surgical navigation.

Registration techniques are categorized into two major types: (1) marker-based [7] and (2) markerfree [8] (Fig. 41.3).

In the marker-based technique, registration requires identifiable markers to be placed within preoperative images that can be easily detected on the patient during the surgery. These markers include dental splints [9], skin adhesive reference markers, and bone-implanted screws [10]. In contrast, the marker-free technique is based on the patient's regional anatomy. This can be performed by registering easily identifiable soft tissue or bony structures on the patient's preoperative scans. Another technique for marker-free registration is laser surface scanning, which is used to match random points on the surface of facial skin to corresponding points on CT or MRI images. More recently, hybrid registration combining methods have been used that combine point registration and surface registration [11].
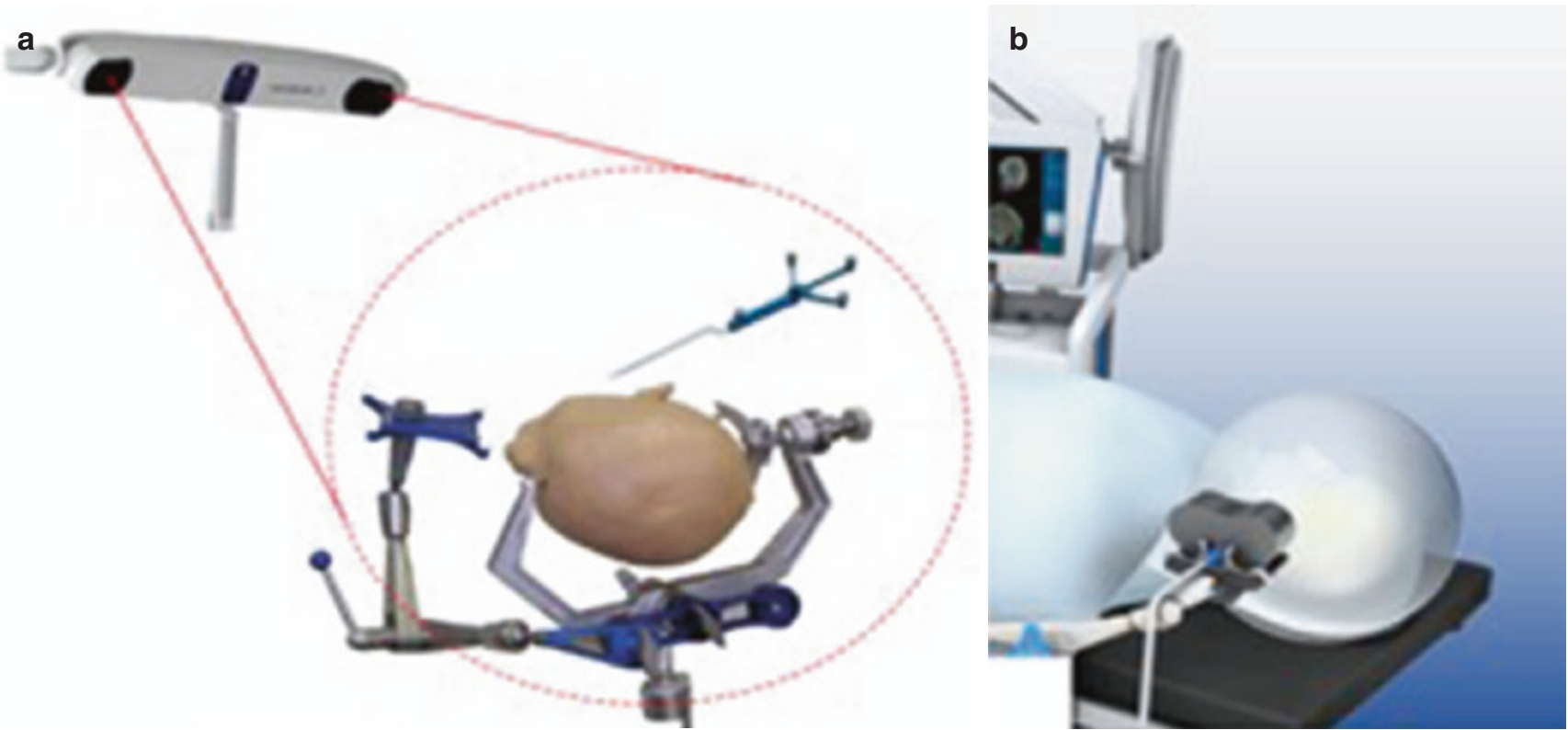

CAssociation of Oral and Maxillofacial Surgeons of India

Fig. 41.2 There are two main types of navigation systems currently available: (a) optical and (b) electromagnetic systems 


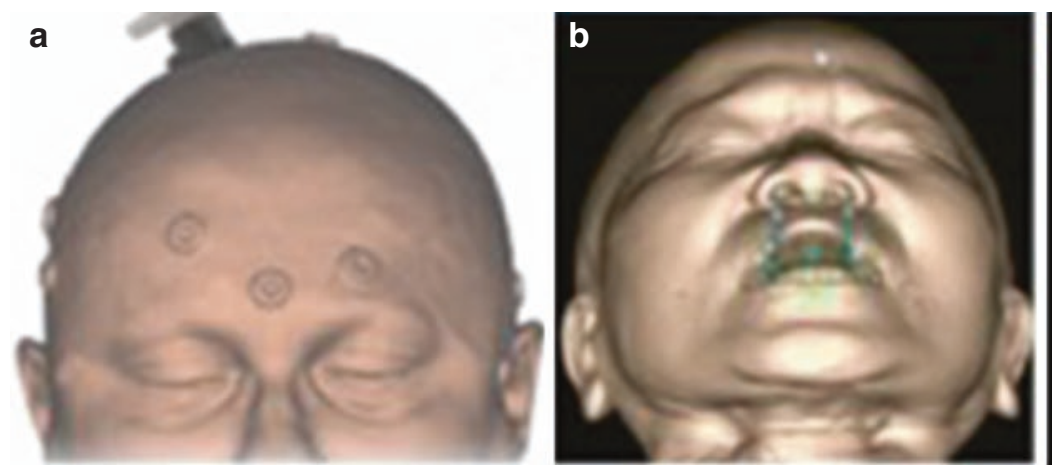

Marker-based registration

(C)Association of Oral and Maxillofacial Surgeons of India

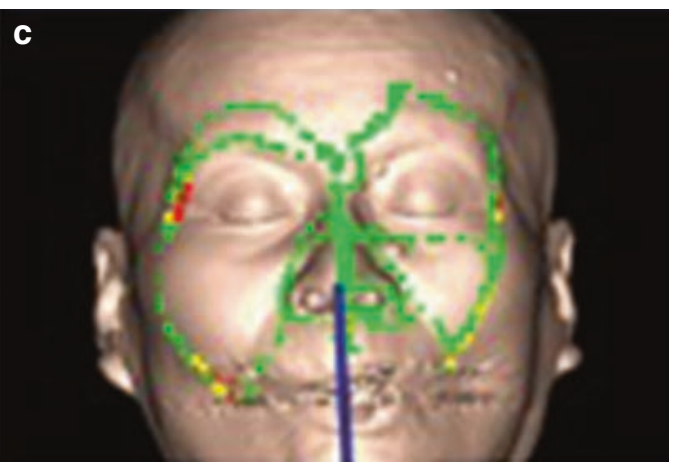

Marker-free registration
Fig. 41.3 Registration techniques for navigation preparation can be categorized into two main groups: marker-based and marker-free. Marker-based registration requires markers that are apparent on preoperative images and that are easily detectable on the patient during the procedure, such as (a) skin adhesive reference markers or (b) a referencing dental splint (c) Marker-free registration relies on the patient's

\subsubsection{Application to Oral-Maxillofacial Surgery}

\subsubsection{Application to the Maxilla and Midface}

The use of navigation systems significantly improves the degree of intraoperative precision and accuracy that can be predictably transferred from the planning stages to the actual surgery. However, navigational accuracy is limited by the type of system used, the method of procuring imaging data, and the intraoperative synchronization of the imaging data with the patient's actual position. The systems currently in use for maxillary and midface surgery are relatively reliable, as most have been modified from their neurosurgical counterparts [12]. Because the maxilla and midface are immovable, unlike the mandible, the position of the skull relative to the reference is stable, and the registration procedure directly reflects this. Therefore, navigation-assisted surgery is the best option at the maxilla and midface.

\subsubsection{Application to the Mandible}

The use of navigation systems is not currently approved for mandibular surgery. This is due to the nature of the mandible to move on its joint (Fig. 41.4), resulting in the registration not being reflected accurately in the navigation of the mandible. However, it may be possible if the position of the mandible is held identical to its intraoperative position during image acquisition.

At present, solutions exist for the application of navigation systems to mandibular surgery. One such option is the use of a dynamic reference frame mounted to the mandible that enables the continuous tracking of mandibular movement during surgery [13]. This method utilizes a sensor frame and mandible/teeth supported markers for the direct tracking of the mandible during surgery. This permits free intraoperative craniomaxillofacial anatomy. Laser surface scanning is applied in a distinct marker-free registration technique, where random points on the facial skin surface are matched to corresponding points on the soft tissues in preoperative computed tomography/magnetic resonance imaging (CT/MRI) images

movement of the mandible. The second option is to maintain an immobile intercuspation position, such as using a maxillamandibular fixation to maintain intraoperative synchronization [14]. Unfortunately, this is impossible to achieve in most intraoral procedures. Therefore, a third strategy has been developed in which the mandible is placed in a reproducible position against the maxilla using an occlusal splint. This method appears to produce no additional error.

\subsection{Clinical Significance in Oral and Maxillofacial Surgery}

There are numerous clinical applications for CAS and intraoperative navigation in oral and maxillofacial surgery. This is reiterated by literature support available over the last decade supporting its use.

\subsubsection{Application for Oral-Maxillofacial Trauma}

Computer-based surgery is a rapidly emerging approach used in some surgical disciplines, and can be used both as a research tool and to improve healthcare. Computer-based surgery, in combination with the use of a navigation system, has been shown to reduce overall operation time in complex anatomic areas, such as maxillofacial trauma surgery (e.g., orbital trauma reconstruction surgery), making surgery more reliable. Using 3D models based on CAS is suitable for preoperative preparation of reconstruction material for bone defects caused by trauma. On the other hand, navigation surgery is most suitable for intraoperative anatomical evaluation [15]. By combining these techniques, safer and minimally 


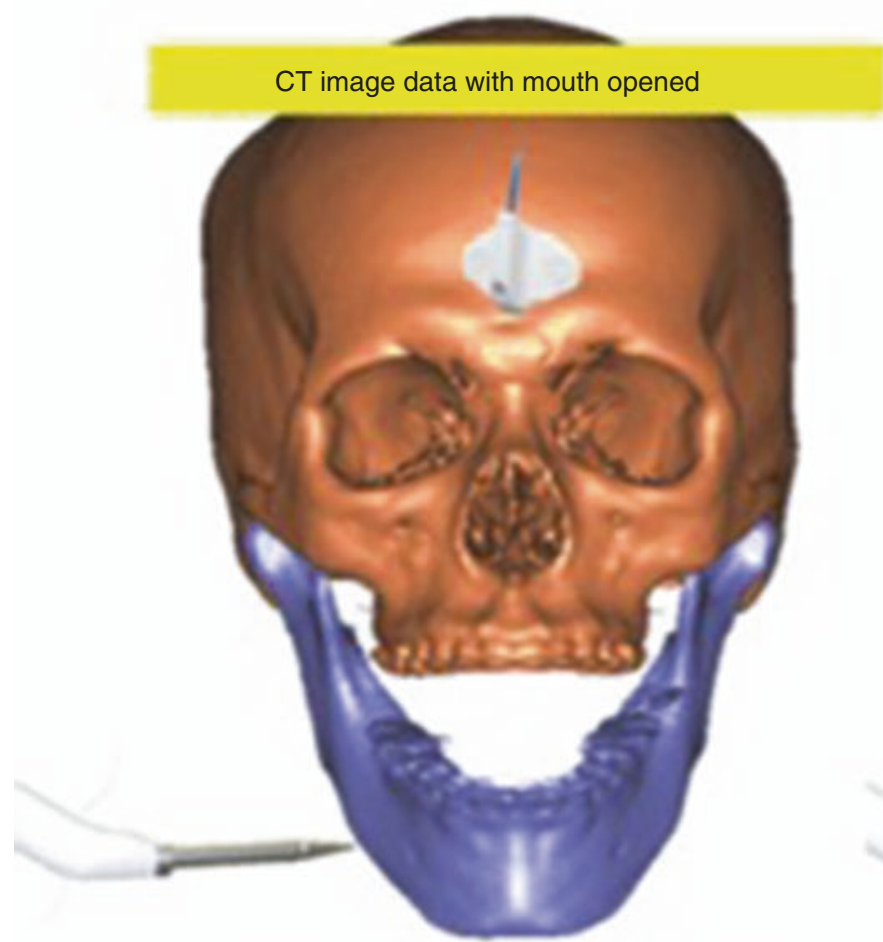

CAssociation of Oral and Maxillofacial Surgeons of India

Fig. 41.4 The positional relationship between the reference and the target becomes unstable due to the free mobility of the mandible. If the mandible were maintained in an identical position during image acqui-

invasive surgery is possible. Maxillofacial trauma may be an important indicator suggesting the use of navigation systems (Figs. 41.5, 41.6, 41.7 and 41.8). Unilateral midfacial and orbital trauma surgery may also be indicators, and are normally treated with open reduction and fixation with navigation assistance.

The largest sample sizes for navigation surgery were found in the field of craniomaxillofacial trauma, offering significant positive results for orbital fracture treatment through navigation-assisted surgery. Midfacial trauma, and particularly unilateral midfacial trauma, was most common example of maxillofacial trauma available in the literature. Some of these reports described the treatment of patients with delayed zygomatic fractures requiring osteotomies to reposition the abnormally healed bones. This results in added complexity for the surgeon due to the lack of known anatomical landmarks. He et al. presented a protocol for the creation of artificial landmarks on the surface of the zygoma [16]. The technique involved the registration being performed with rigidly fixed, light-reflecting spheres placed on the patient's skull. Subsequent soft tissue surface scanning was performed using a laser pointer to complete the process.

Another challenging facet of oral and maxillofacial surgery is the management of orbital fractures. This can prove

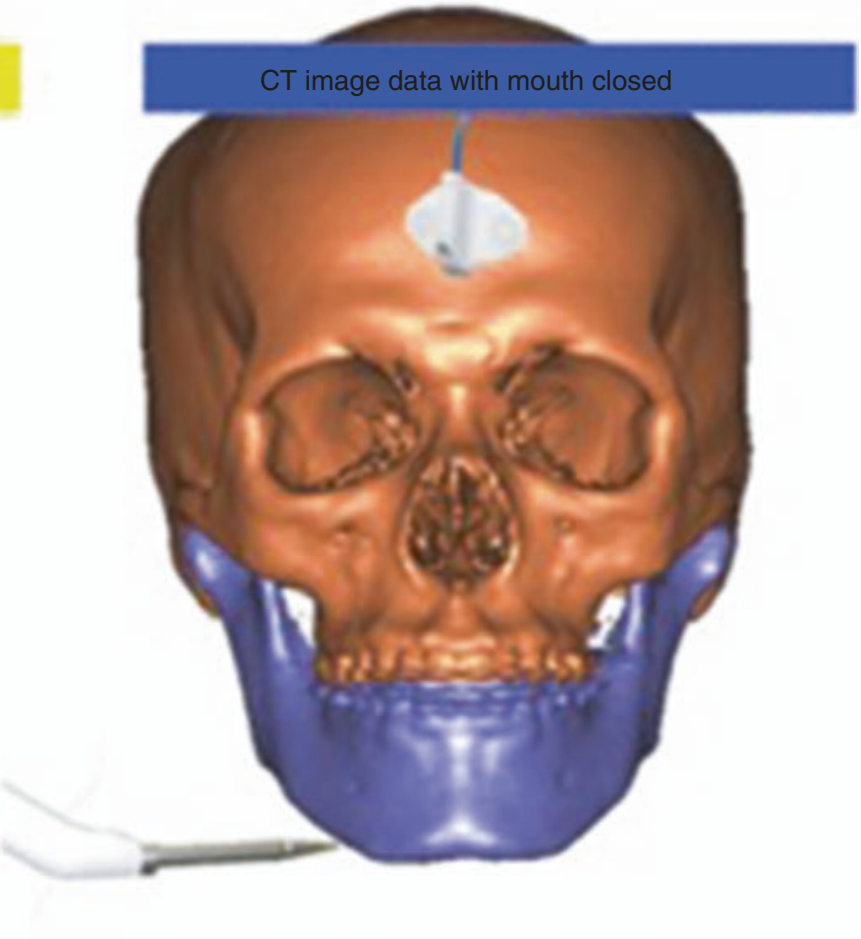

sition and the surgical procedure, then all structures within the image could be fixed in an identical position, thereby ensuring the accuracy of the navigation surgery

to be demanding even for the most experienced of surgeons. Literature indicates that intraoperative navigation is a very useful tool in post-traumatic orbital reconstruction. Another rising trend is the use of patient-specific implants (PSIs). A recently published literature also discusses the use of PSIs with navigation guidance [17]. A control group was treated with navigation using prebent titanium mesh. Several significant factors were reported favoring the study group. PSIs are poised to make a significant change in the management of orbital trauma.

Innovations such as intraoperative navigation and computer-assisted surgical planning have been shown to improve the efficacy, accuracy, and predictability of surgical procedures. The 3D reconstruction abilities of software could be used to virtually display the patient's anatomy throughout the case, allowing for stereotactic navigation. During the surgery, the intraoperative navigation system helps surgeons to control the positions of implants or of repositioned bone, and assists in verifying the final location. A navigation system enhances the surgeon's ability to measure the extent of resection and to confirm the orientation of bone grafts used for reconstruction. Using this approach, it is possible to minimize the human error factor by increasing the adherence to a pre- 

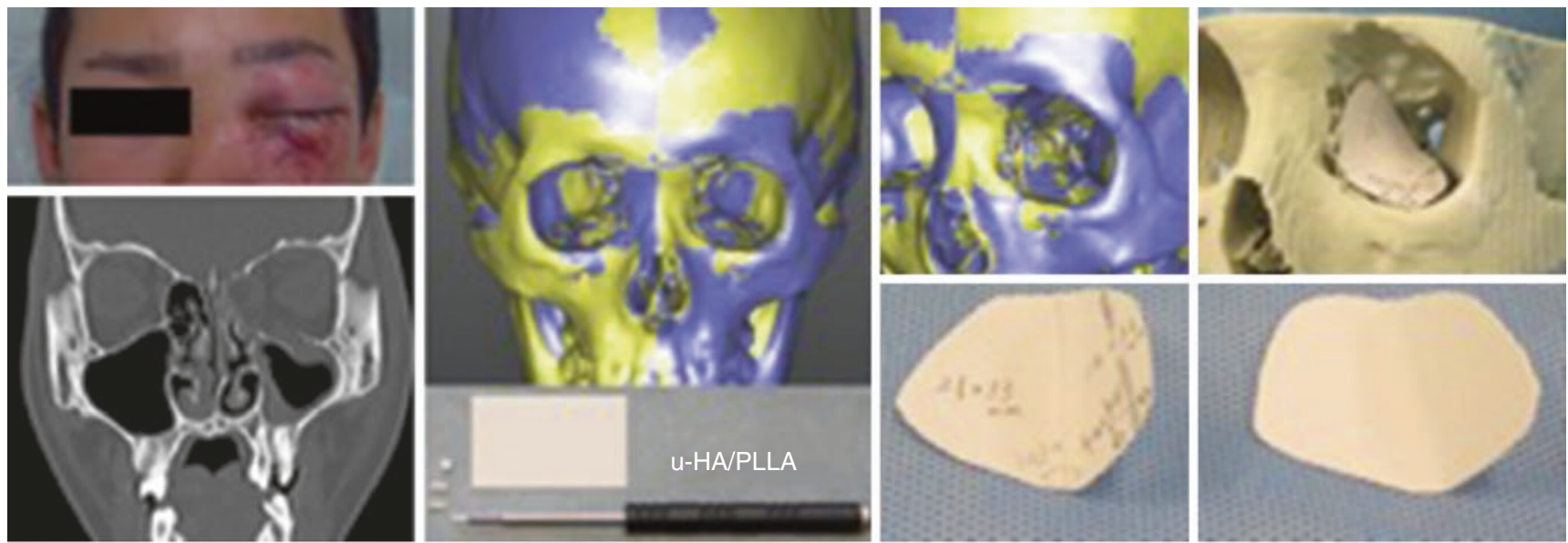

CAssociation of Oral and Maxillofacial Surgeons of India

Fig.41.5 A 17-year-old male undergoing a large orbital floor to medial orbital fracture reconstruction. Surgeons first created a threedimensional model that was mirrored by CAS, and determined the angle and form of reconstruction material using third-generation bioac- tive/bioresorbable materials, the SuperFIXORB-MX® (OsteotransMS®) system; TEIJIN Medical Technologies Co., Ltd, Osaka, Japan, according to the shape of the orbits to be reconstructed
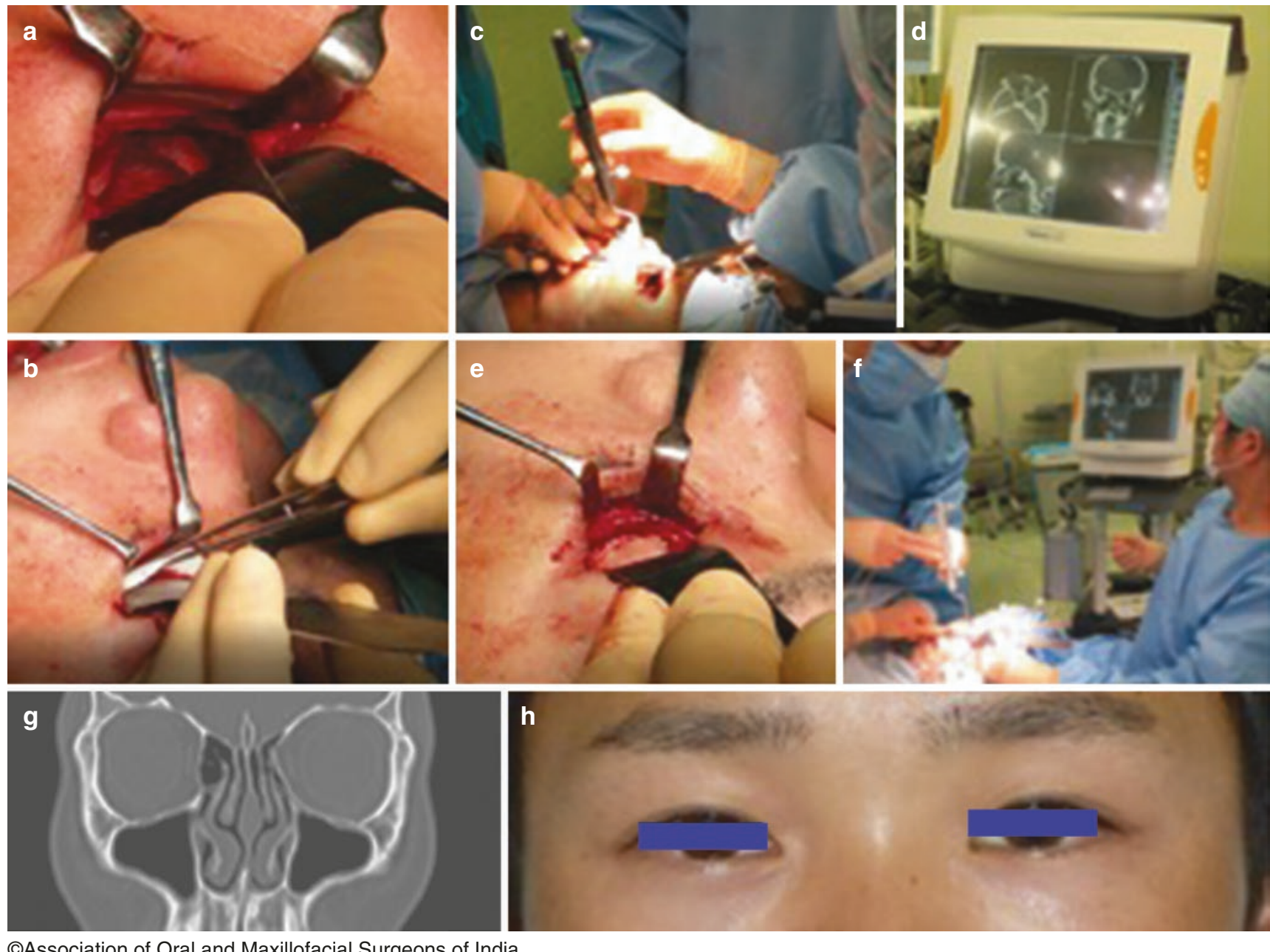

CAssociation of Oral and Maxillofacial Surgeons of India

Fig. 41.6 (a-h) A 17-year-old male undergoing a large orbital floor to medial orbital fracture reconstruction. Navigation will determine the exact intraoperative anatomical form. Reconstruction material before
CAS was positioned at the reconstruction site. We then confirmed the exact position of the reconstruction material and reconstructed the orbital shape using the navigation system 

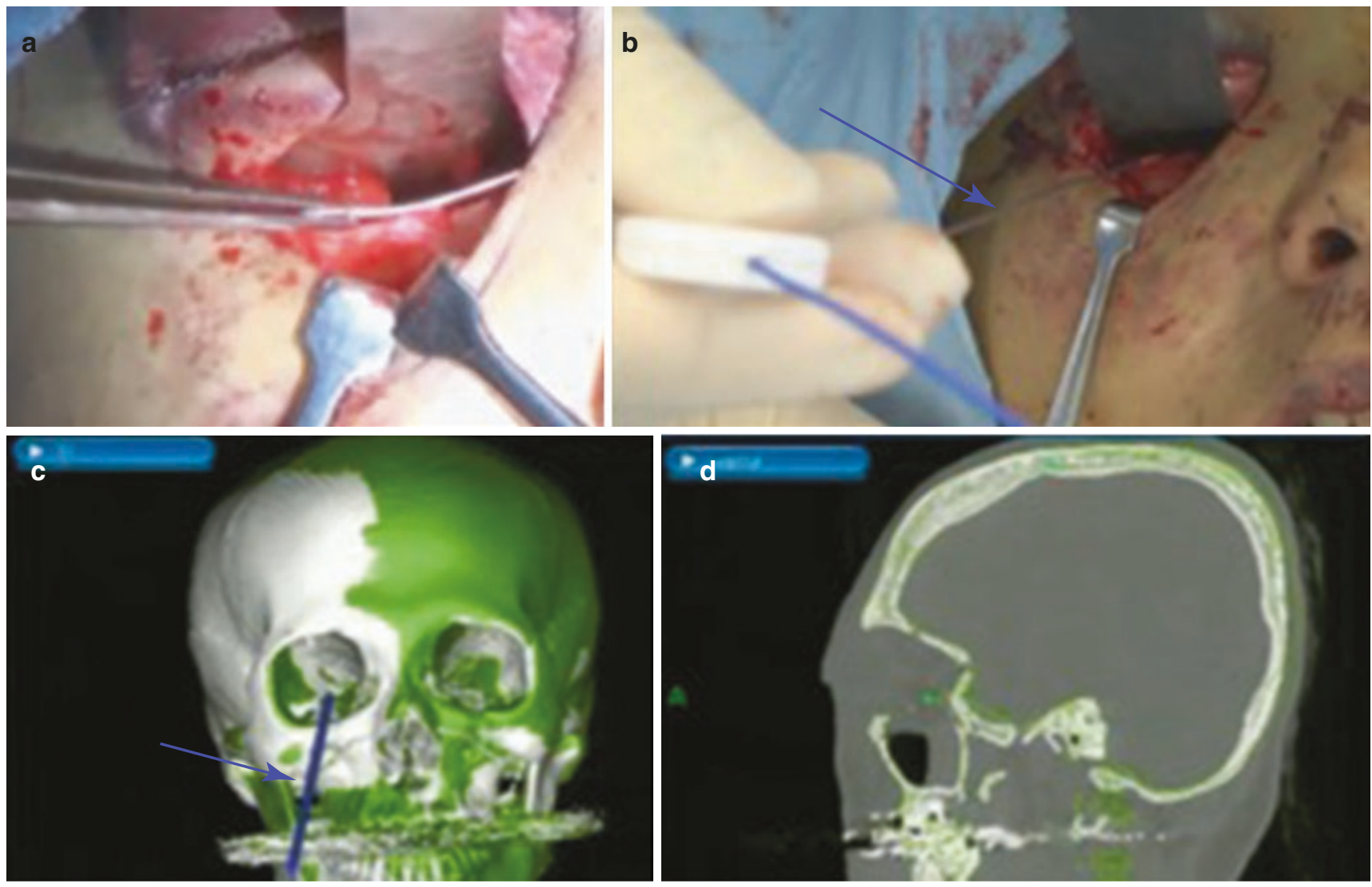

CAssociation of Oral and Maxillofacial Surgeons of India

Fig. 41.7 (a) The bioresorbable mesh plate was positioned to support the orbital globe. (b) The reconstructed site was confirmed to match the mirror image using a tip pointer with a navigation system. (c) Intraoperative navigation system screenshot showing a multiplane view of the position of the surgeon's navigation probe in relation to the orbital floor defect region at the time of localization. (d) Shows the reconstruction plan image, created using the mirroring technique
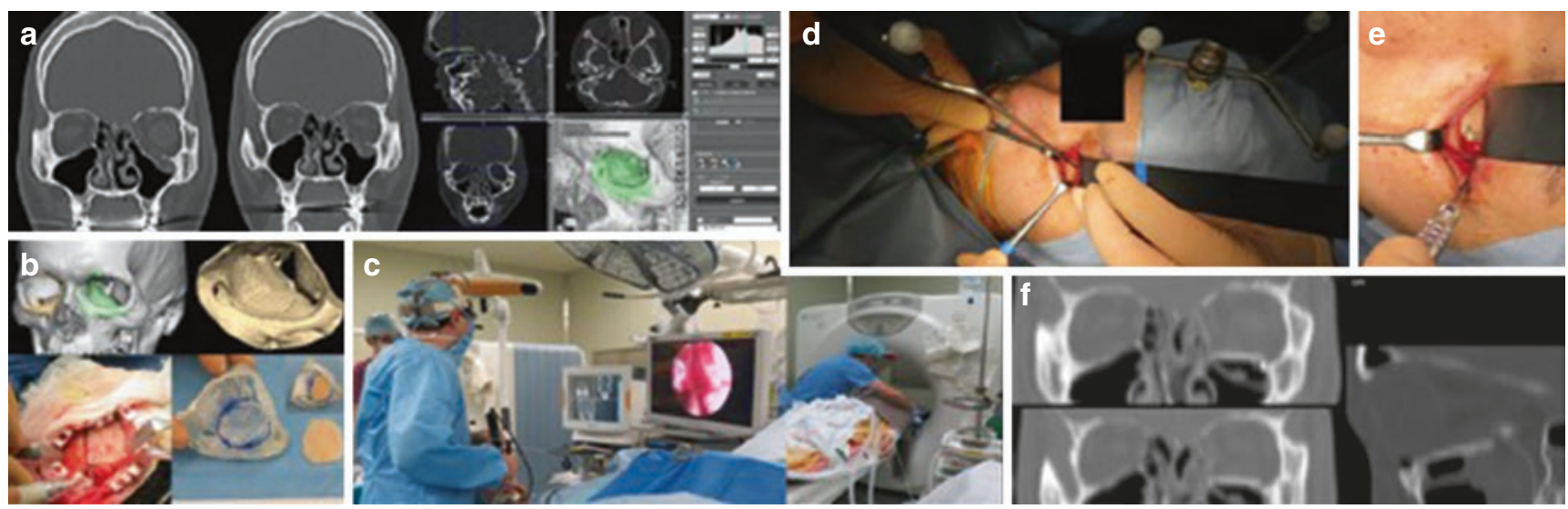

CAssociation of Oral and Maxillofacial Surgeons of India

Fig. 41.8 (a, b) A 33-year-old male undergoing a nasoorbitoethmoidal (NOE) and maxillary fractures open treatment, including a large orbital floor to medial orbital walls fractures reconstruction. We, maxillofacial surgeons, first created a three-dimensional (3D) precise preorbit to orbital groove model that was mirrored by computer-assisted simulation, and determined the anatomical form of reconstruction for autogenous bone harvested from calvaria using Piezosurgery ${ }^{\circledR}$ according to the 3D shape of the orbits to be reconstructed (produced by Yasojima Proceed Co., Ltd. Osaka, Japan). Navigation will determine the exact intraoperative 3D anatomical form. (c) The fractured deep medial orbital wall was precisely reduced under an endoscopic-assisted navigation-guided surgery by otorhinolaryngology-head and neck surgeons. (d, e) We then reconstructed the large orbital floor and confirmed the exact position of the autogenous calvarial bone for accurate reconstruction of the orbit using the navigation system, followed by fixation using titanium screws. (f) The use of intraoperative CT in the hybrid operation room can provide rapid $\mathrm{CT}$ data during surgery for the final 3D confirmation of complex orbital reconstruction cases 
operative plan. Furthermore, this also helps in reducing the incidence of postoperative complications due to improperly positioned or oriented bone grafts, plates, or fixation screws. Virtual surgical planning (VSP) combined with 3D printing technology has improved surgical efficiency and precision through the generation of 3D surgical models, implants, and guides [18]. This increases the number of indicators suggesting the use of VSP by the surgeon, as it offers additional tools in preoperative planning and intraoperative decision making. Both VSP and 3D models may be used to plan the optimal reconstruction material in terms of the volume, shape, and dimensions required. They can also be used to produce templates for resective surgery to accurately demarcate the boundaries for resection and/or to plan more efficient and accurate reconstructive strategies. A further advantage of using 3D models is a reduction in total operative time and the elimination of potential complications from prolonged surgery.

\subsubsection{Application for Oral-Maxillofacial Tumor/Cancer}

Computer-assisted navigational surgery is a proven method for reducing operating time and increasing reliability in complex surgical procedures of the infratemporal fossa and the periorbital region [19] (Fig. 41.9). Navigation-assisted surgery was introduced in the field of oral, head, and neck tumors more than 20 years ago. The use of navigation in the management of benign and malignant lesions is discussed later.

Malignant lesions of the head and neck have a high rate of recurrence (25-48\%). Tumor control essentially depends on the extent of the tumor, its location, and the margins of resection, with the latter being a very important prognostic factor in cancer surgery. Feichtinger et al. [20] used navigation systems to evaluate the resection margins in the treatment of six patients with carcinomas in the nasal cavity, maxillary sinus, and oral cavity. In four patients, further resection had to be performed after an assessment with a navigation system
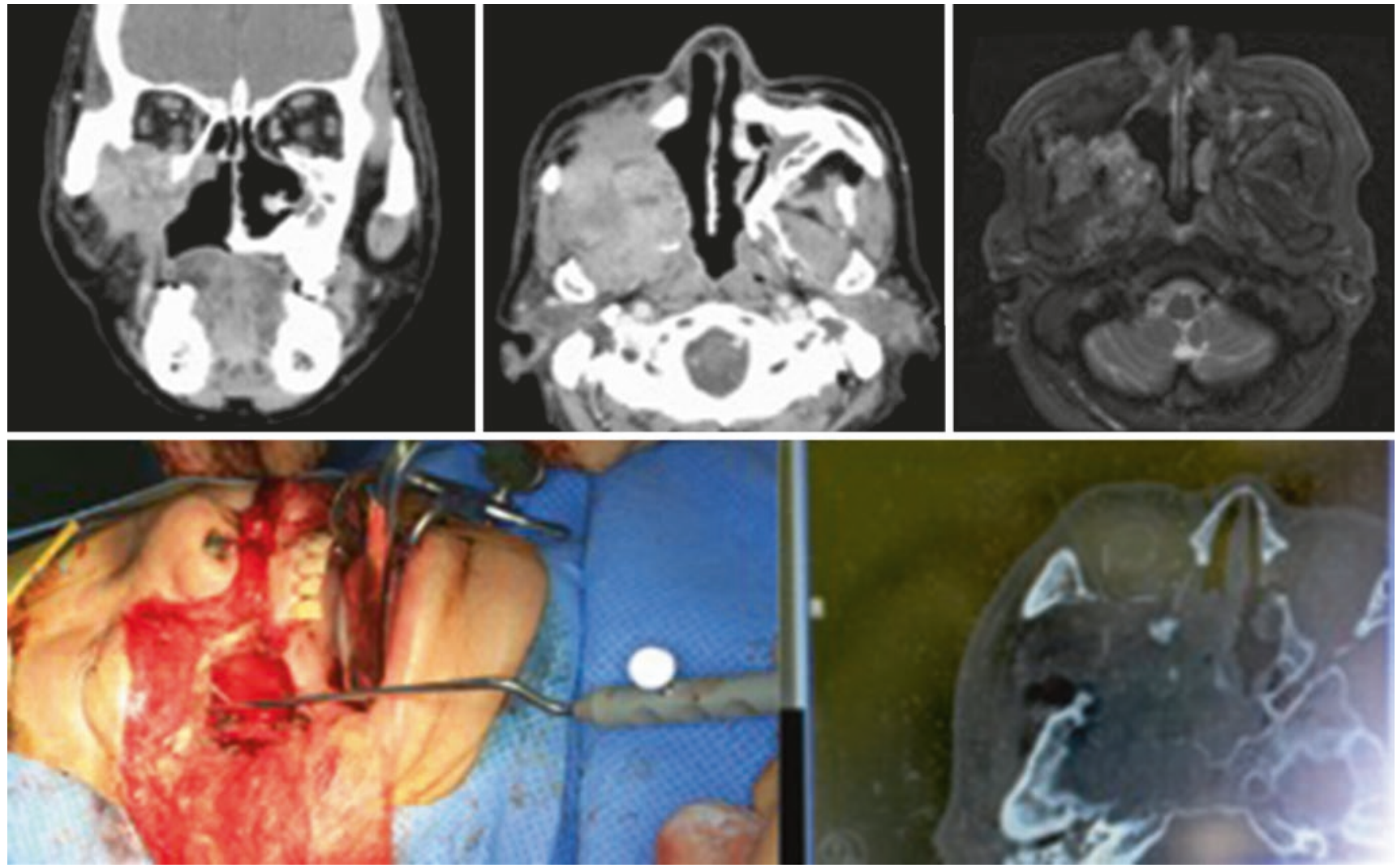

CAssociation of Oral and Maxillofacial Surgeons of India

Fig. 41.9 A 79-year-old male with recurrent ameloblastoma of maxilla-infratemporal fossa. Because of the complexity of the local anatomy, tumors in the infratemporal fossa present a challenge to oral and maxillofacial surgeons. Recurrent malignant tumors in this area are particularly difficult to resect because scars from previous operations may dislocate important structures. A navigation technique has been introduced to resect infratemporal fossa tumors and was successfully applied for the resection of recurrent malignant tumors. The visible navigation during surgery could increase the accuracy and safety of the operations and enhance surgeon confidence 
using positron emission tomography (PET)-CT scans proved the initial resection to be unsatisfactory. This demonstrates that navigation surgery based on PET-CT image fusion is an excellent tool for improving the local control of advanced head and neck cancer.

Navigation-assisted surgery has also been successfully used in the management of benign tumors of the maxillofacial region [21]. This was performed with features such as mirroring and side-to-side comparisons, and was applied with successful results. Use of customized hydroxy-apatite prosthesis with pre-embedded titanium implants was used for reconstruction. Optimal positioning is secured using navigation. Some interesting techniques for reconstruction are discussed now.

It is important to remember that navigation is an excellent tool when it comes to its use in the skull base [22], the midface [23], and the neck [24]. However, its use in mandibular tumors is limited due to the possibility of changes in the spatial orientation of the lower jaw, as described earlier.

\subsubsection{Application for Orthognathic Surgery}

The primary determinants of success in orthognathic surgery include an accurate diagnosis, meticulous treatment planning, and the ability to transfer the plan precisely to the patient intraoperatively. In earlier years, this was performed by a process of model surgery in a laboratory using articulators on plaster casts, which were then transferred to the patient using acrylic splints during the surgical procedure (Figs. 41.10 and 41.11). The procedure was error prone at multiple levels within the sequence. Literature reports an error of up to $5 \mathrm{~mm}$ using this type of treatment sequence. With the advent and routine usage of CAD/CAM technology
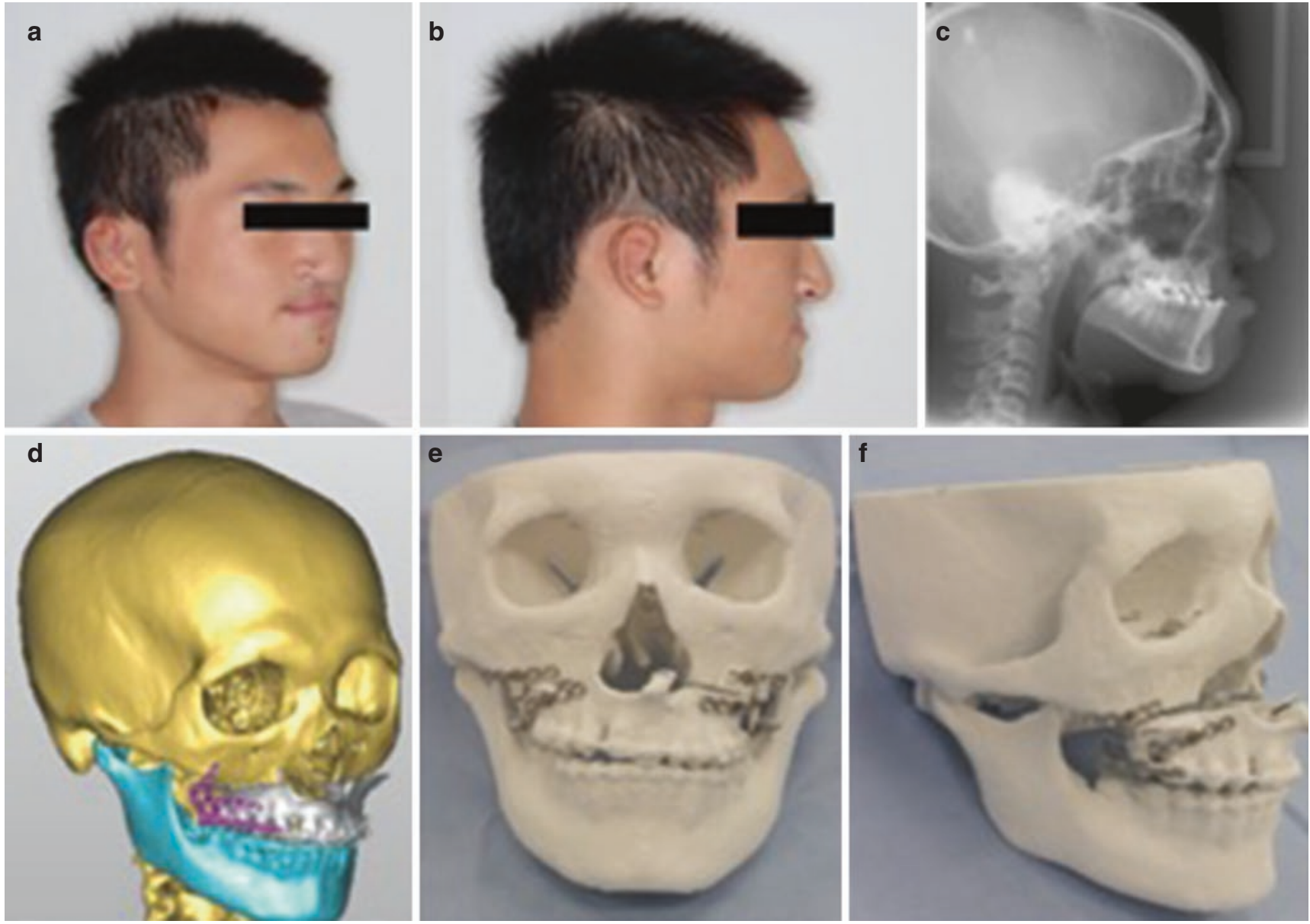

CAssociation of Oral and Maxillofacial Surgeons of India

Fig. 41.10 Optimal treatment planning and preparation using 3D models. CAS for cleft lip and palate-related severe maxillary hypoplasia deformity patients using maxillary distraction osteogenesis. (a-c) A 17 year old male undergoing maxillary distraction for cleft-related midfacial retrusion. (d) Detailed computer simulation for maxillary distrac- tion surgery in a patient with severe maxillary hypoplasia deformity was used to determine the optimal treatment plan, such as the distraction direction and degree of advancement. (e, f) A 3D printed skeletal model was used to prepare for a maxillary semi-custom-made distractor setting with fabrication 

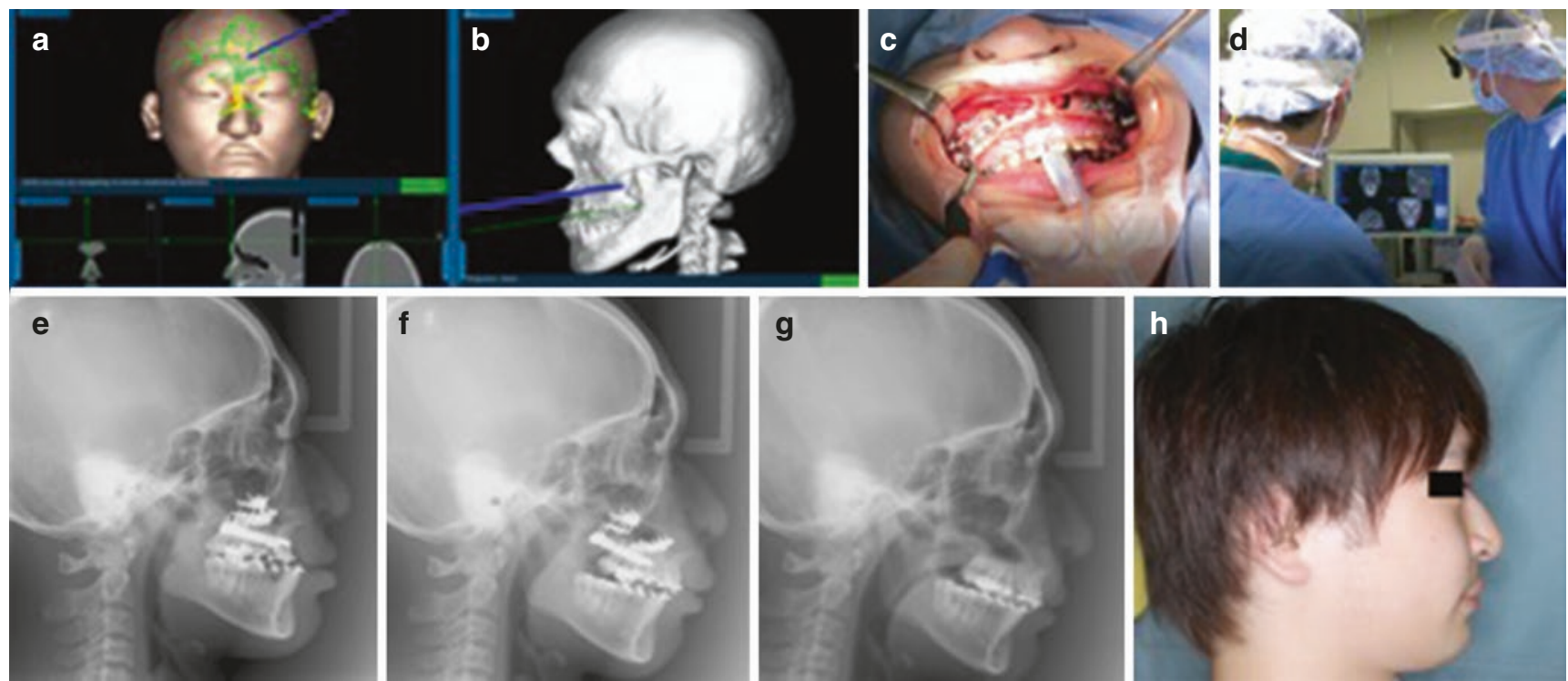

CAssociation of Oral and Maxillofacial Surgeons of India

Fig. 41.11 A 17-year-old male undergoing maxillary distraction for cleft-related midfacial retrusion. (a-d) Accurate transfer of the treatment plan to the patient in the operating room was acheived. Using the navigation system, precise placement of the maxillary distraction planned pre-

and virtual surgical planning, the workflow of treatment planning in orthognathic surgery has undergone a paradigm shift. The conventional workflow of model surgery and splint fabrications is slowly paving the way for VSP and custom printed 3D splints and guides.

Although the degree of inaccuracies associated with treatment planning is reduced using this approach, certain drawbacks such as autorotation of the mandible and lack of control on the vertical position of the maxilla are still prevalent.

There are three methods by which CAS is used in the practice of orthognathic surgery: (1) using real-time intraoperative guidance with surgical navigation for the repositioning of the maxilla and mandible; (2) use of 3D printed cutting guides for precise osteotomy and repositioning, with or without customized 3D printed osteosynthesis plates; and (3) the use of wafer-less surgical planning where the printed implant doubles as both a cutting guide for the osteotomy and as the fixation devices. Many clinical studies have evaluated the efficacy of these methods with promising results.

Mazzoni et al. were the first to report the use of intraoperative navigation in orthognathic surgery, in 2010 [25]. They calculated the overlap error to assess the accuracy of operatively at the set position was performed. The surgeon then confirmed the same distraction direction that was preoperatively planned by the intraoperative navigation. (e-g) Shows the radiographs during and post treatment. (h) Shows the final post surgical result of the patient

the technique after surface matching the virtually planned model and the postoperative CT scan. The accuracy was reported for the entire facial skeleton, rather than for each component individually (maxilla, mandible, chin), with a mean match error for each patient ranging from 0.28 to $1.99 \mathrm{~mm}$. Repeatability $(<2 \mathrm{~mm})$ in the face area ranged from $77.5 \%$ to $96.2 \%$ between patients, with a mean reproducibility of $86.5 \%$.

Zinser et al. published a clinical controlled trial study in 2013 that compared the navigation technique with conventional technique, using 3D surgical guides and intermaxillary splints [26]. The highest accuracy for transfer of the maxillary plan to the patient was observed when a 3D surgical guide was used, and no significant linear differences between the planned virtual model and the postoperative results were present in any direction. The navigation technique only showed a significant mean linear difference in the vertical dimension, and differences in angulation were not significant in either group. In contrast, significant linear differences were shown for the classic intermaxillary splints between the planning results and the actual results in the sagittal and vertical dimensions. Differences in plane angulations when using an interocclusal splint were also significant. The usefulness of navigation-assisted surgery for orthognathic surgery was supported based on previous reports.

Published literature demonstrates that all studies have met the 2-mm success criterion, which refers to a maximum difference of $2 \mathrm{~mm}$ between the virtual planning performed and 
the actual surgical outcome. Zinser et al. reported the only prospective controlled clinical trial comparing CAD-CAM splints, navigation surgery, and intermaxillary splints for the transfer of maxillary planning [26]. The CAD-CAM splints, patented by the authors, were used for maintaining the mandibular condyles (TMJ) in their centric relation. Mandibular positioning still poses a challenge during orthognathic surgery, with none of the solutions having attained "goldstandard" status.

An important clinical limitation for the use of navigation is the increase in operating time [27]. Though there is ample support for increased accuracy levels with the use of navigation, the prolonged operating time still remains a deterrent for the routine use of intraoperative navigation in orthognathic surgery. A recent study indicates that dynamic navigation systems have an entry error of approximately $0.4 \mathrm{~mm}$ [28] and an angular deviation error of approximately $4^{\circ}$ [29]. Further technological developments can be expected in the field of orthognathic surgery, and an appropriate update for surgeons is required.
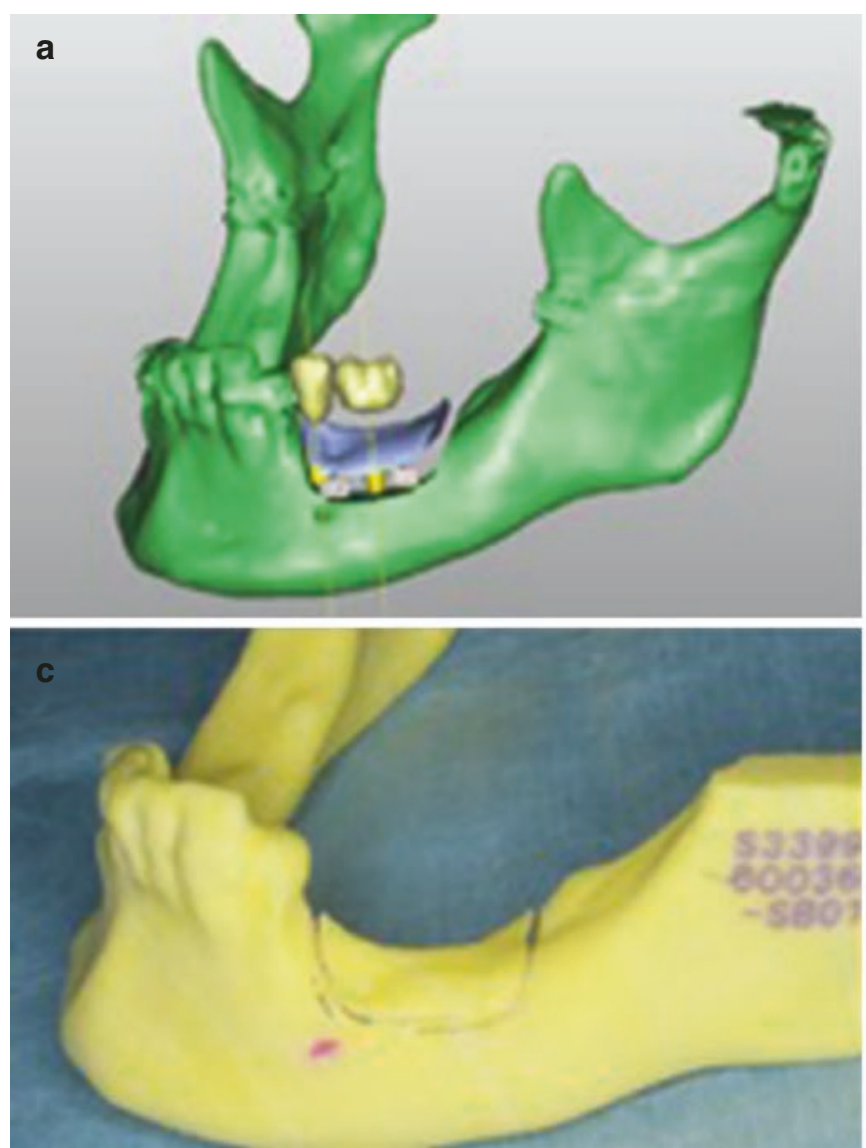

CAssociation of Oral and Maxillofacial Surgeons of India

Fig. 41.12 (a-d) Computer simulation based on preoperative CT data will determine the bone augmentation method for implantation. In this case, we chose bone augmentation using the sandwich technique. The necessary bone augmentation volume and position of the anatomically

\subsubsection{Application for Preimplant Bone Augmentation/Dental Implants}

Loss of teeth and supporting structures is a common occurrence in the field of oral and maxillofacial surgery for patients who have suffered extensive alveolar bone defects caused by cysts, tumors, facial trauma, or severe periodontal disease [30]. Comprehensive reconstruction methods, including rehabilitation with multiple dental implants, are commonly required to restore function in these patients.

Issues like atrophic jaws, where dental implants cannot be placed due to the loss of bone corpus, are managed by regenerative procedures. Although bone augmentation at the mandibular posterior area is important for occlusion reconstruction using dental implants, it is difficult due to anatomical limitations arising from the inferior alveolar nerve (IAN) and the mandibular bone. However, bone augmentation at the mandibular posterior area can be performed safely and reliably using CAS with 3D modeling and navigation systems (Figs. 41.12 and 41.13).
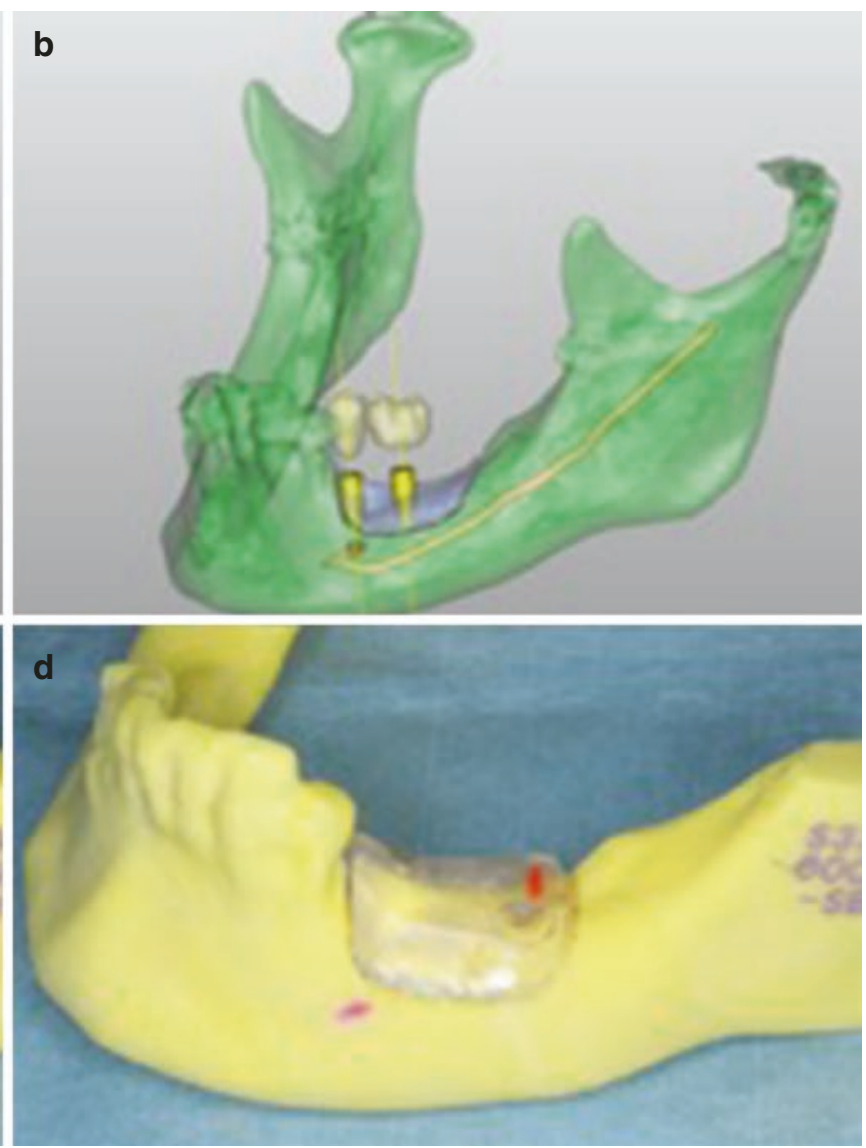

important inferior alveolar nerve (IAN) were confirmed using computer simulation. Based on this information, we will determine a safe bone cutting line. Surgeons then created a surgical guide using a 3D model based on simulation 

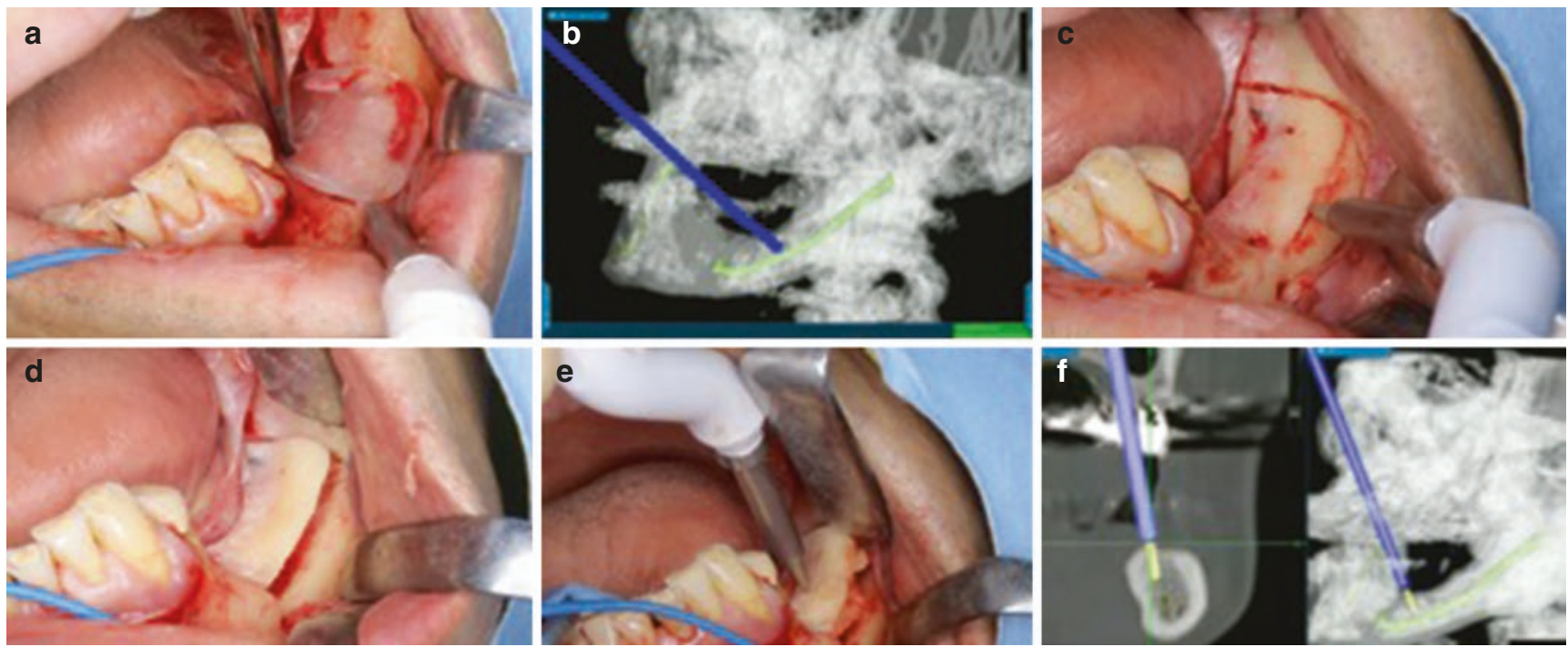

CAssociation of Oral and Maxillofacial Surgeons of India

Fig. 41.13 (a-d) This is a continuation of Fig. 41.12. While confirming the position of the IAN in real time based on intraoperative navigation, the surgeon cut the alveolar bone with reference to the surgical guide created before surgery. (e) The surgeon then inserted autologous

Traditionally, implants have been placed freehand or with the use of laboratory-fabricated guidance stents. The use of CT-guided 3D printed stents with coordinated drill sequencing has minimized errors to a level of less than $2 \mathrm{~mm}$ for crestal and apical deviations, and to less than $5^{\circ}$ in angulations. Dynamic navigation systems use a time-effective method to accurately place implants with equivalent implant placement error. Currently the Image-Guided Implantology system (Navident; HERMANS Corp., Tokyo, Japan) is the only dynamic image navigation system (DINS) available for in-office dental procedures in Japan. Its counterpart in the United States is the passive optical dynamic navigation in implantology (X-Nav Technologies, Inc., Lansdale, PA, USA).

Navigation in dental implantology can add the following advantages: (1) precise depth control and reduced risk of IAN damage [31]; (2) help in planning for flapless surgery or limited flap elevation surgery with reduced postoperative morbidity [32]; and (3) accurate spacing and implant angulation compared to using a freehand approach.

The use of virtual implant planning and intraoperative navigation allows for prosthodontic and surgical coordination due to its planning accuracy and implementation in actual surgical scenarios. bone taken from the donor and increased the alveolar bone vertically. The most important goal in this surgery is to acquire alveolar bone height as planned before surgery. (f) The bone height can be confirmed in real time based on intraoperative navigation
Dynamic navigation methods have similar advantages, including high accuracy, time- and cost-effectiveness, minimally invasive techniques, and flexibility in changing the implant size, system, and location during the surgical procedure [33]. An additional advantage is comfort provided the surgeon in the form of posture, facilitating reduced bending of the neck and back. For example, dynamic navigation allows implant placement for patients with a limited mouth opening, or requiring an implant at a second molar site with reduced access, by relying on a navigation screen to guide the drill sequence without direct visualization of the patient's mouth.

\subsubsection{Clinical Applications for the Removal of Foreign Bodies}

Retrieval of foreign bodies in the craniomaxillofacial region is often extremely dangerous due to the proximity to various vital structures within a limited anatomical space [34]. This may be made more complex by the presence of deep foreign bodies secondary to severe trauma such as gunshot wounds or blast injuries, which significantly alter the anatomy. Precise location of the foreign body is the first step in the retrieval process, and this may be accomplished with preoperative scans and 3D image rendering. The intraoperative step is next, and is more challenging, as the exact location of the foreign body within the surgical field must be ascertained. Traditional methods utilize a stereotactic "double needle" method with venipuncture needles for triangulation 

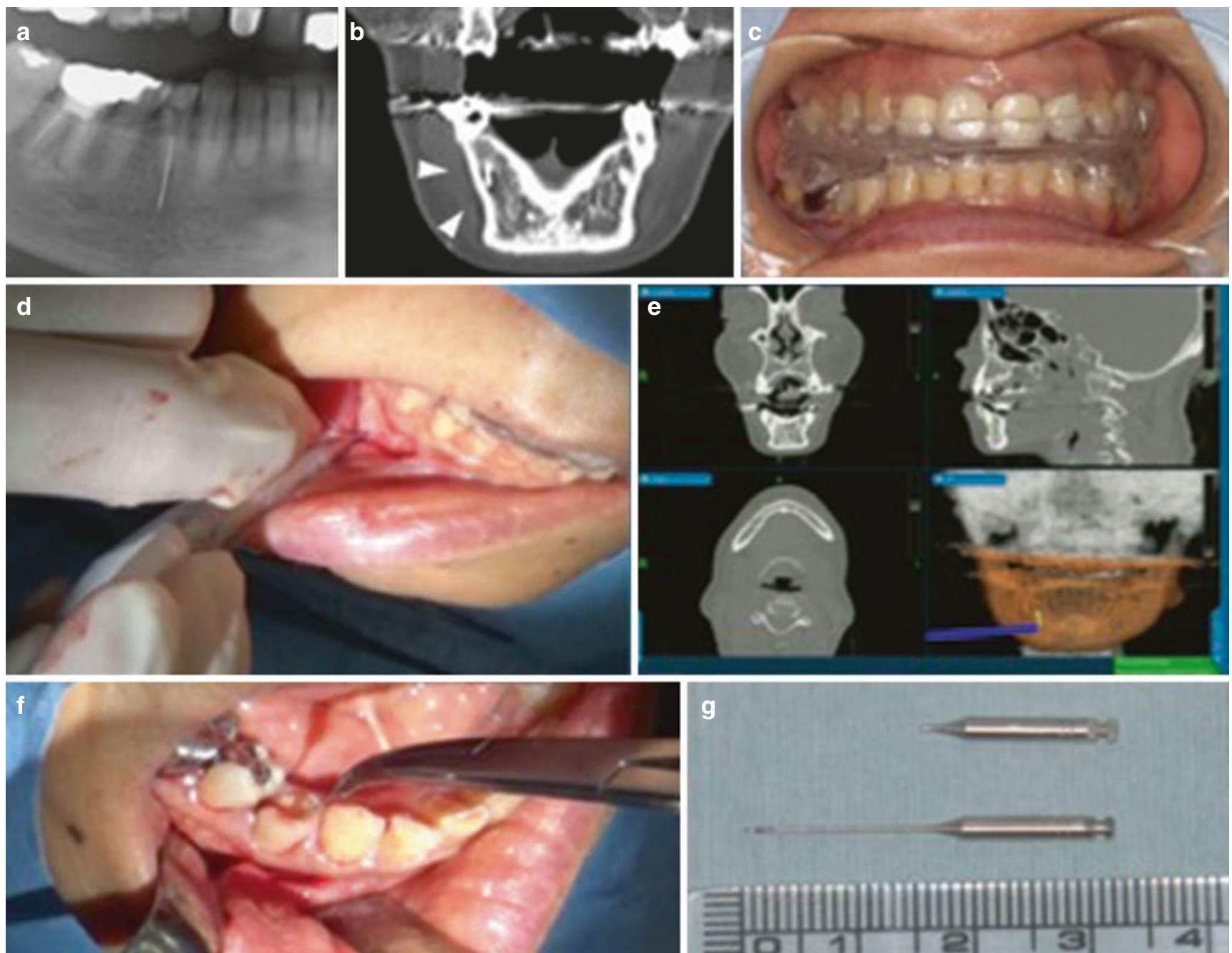

CAssociation of Oral and Maxillofacial Surgeons of India

Fig. $41.14(\mathbf{a}-\mathrm{g})$ A 65-year-old female with a foreign body due to dental instrument breakage in the mandible, occurring during an endodontic treatment for a restorative dental procedure. $(\mathbf{a}, \mathbf{b})$ Fracture of root canal instruments, with a fractured piece protruding beyond the apex, is a troublesome incident during endodontic treatment. Locating and retrieving these objects represents a challenge for maxillofacial surgeons because they are difficult to access due to the proximity between the foreign body and vital structures. Using the navigation system for mandible treatment is difficult as the mobile nature of the mandible complicates its synchronization with the preoperative imaging data during surgery. (c) The broken dental instrument was removed using a minimally invasive approach with a surgical navigation system and an interocclusal splint for stable, identically repeatable positioning of the mandible. (d, e) Based on the 3D position of the navigation probe, a location that best approximated and the most anterior extent of the fragment was selected. (f) A minimal vestibular incision was made at this location, a subperiosteal reflection was performed, and the foreign body location was confirmed using the navigation system. (g) The instrument was carefully visualized and extruded from the apical to the tooth crown side and was then removed using mosquito forceps through the medullary cavity of the crown side of the tooth of the foreign body using plain radiographs [35]. This involved the two reference needles being sequentially placed until both met the foreign body on radiographs. Through blunt dissection, one of the needles is made to contact the foreign body and locate it. It is important to understand that it may be difficult to distinguish small changes in position on plain radiographs. The use of $\mathrm{C}$-arm digital fluoroscopy was a significant advancement for this method, providing rapid radiography [36]. However, as with plain films, fluoroscopic images are 2D, which imposes limitations when locating objects in a 3D space. The use of intraoperative ultrasound imaging has also been proposed to localize foreign bodies. However, the precision involved in positioning may be questionable, and its use in the oral cavity may also be limited by the size of the instrument.

Intraoperative navigation systems could allow a foreign body to be accurately located in 3D space; these systems are very effective for removing a foreign body during facial surgery [37] (Figs. 41.14 and 41.15). One limitation of navigation is the incapability of the system to account for 

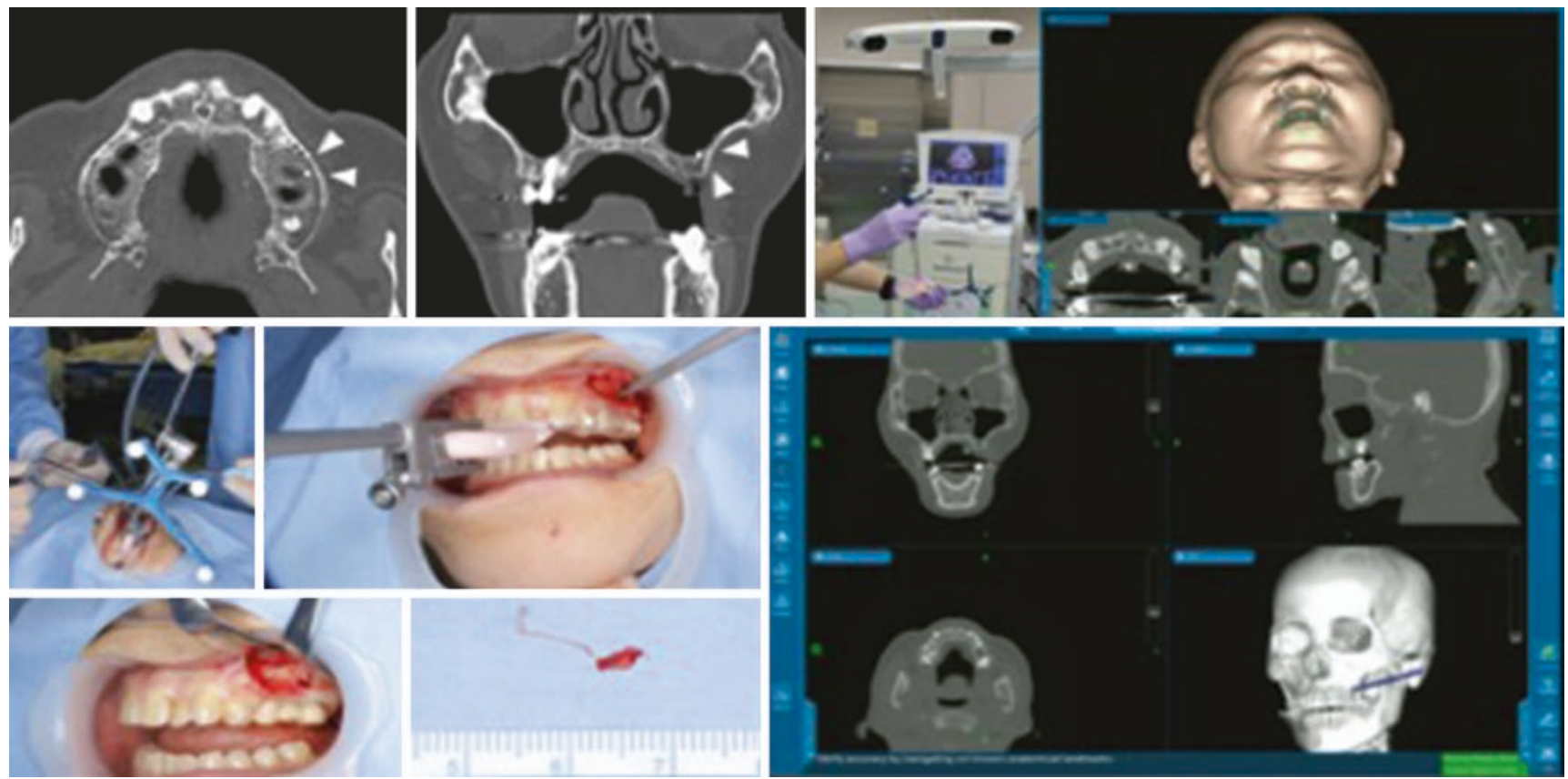

CAssociation of Oral and Maxillofacial Surgeons of India

Fig.41.15 A 78-year-old female with a small foreign body in the maxilla. Accurate determination of the position of the foreign body in the maxillofacial region can be challenging. This may be due to a smallsized foreign body or a limited inflammatory response. Navigation systems are helpful in identifying the location of the foreign body, determining the optimal approach, and performing the surgical proce-

intraoperative soft-tissue changes. The use of navigation techniques superficial tissues or to minimize soft-tissue manipulation may help us overcome this to an extent.

\subsubsection{Application for Dentoalveolar Surgery}

Dynamic computer-based image navigation technology is a good method for increasing accuracy while minimizing the invasiveness of surgery. It has the additional advantage of real-time access to intraoperative radiographs, which enable us to perform complex dentoalveolar procedures with relative ease. Currently, the Image-Guided Implantology system (Navident; HERMANS Corp.) is the only dynamic image navigation system (DINS) available for in-office dental procedures in Japan. This is an ultraviolet-based optical system, which was approved by the US Food and Drug Administration for the placement of dental implants. However, its use for other dentoalveolar procedures is not approved. Its advantages in the field of dental implantology have already been discussed earlier. The use of dynamic navigation when applied to complex dentoalveolar procedures, such as the surgical removal of third molars, is similar to its use for dental dure using a minimally invasive surgical strategy. In this case, registration could be reliably performed before surgery using an optical navigation system that facilitates the process, utilizing splints with embedded reference points. This method can decrease the operation time

implants, helping the surgeon prevent inadvertent damage to key structures like the IAN, roots of adjacent teeth, or the lingual plate of the mandible [38]. Common indications for the use of navigation in dentoalveolar surgery include third molar extractions and the location and extraction of supernumerary [39] or malposed teeth (Fig. 41.16), as well as teeth, which get iatrogenically displaced into the sinus, the sublingual pouch, or even the infra temporal fossa.

The use of CAS in oral and maxillofacial surgery has been enhanced with the increasing availability of CBCT. This in turn has promoted the use of Dynamic Image Navigation. As described earlier, the use of DINS during surgical extraction improves visualization of the regional anatomy, preventing or minimizing complications secondary to dentoalveolar surgery. In addition, improved instrument control allows for reduced bone removal, minimization of the surgical access size, and an overall reduction in the morbidity of the procedure. As discussed earlier, this technology also allows for improvement of the ergonomics involved during the surgery. Lastly, dynamic guidance can serve as an effective teaching tool for young surgeons by displaying the surgery and the locations of vital structures on screen. 


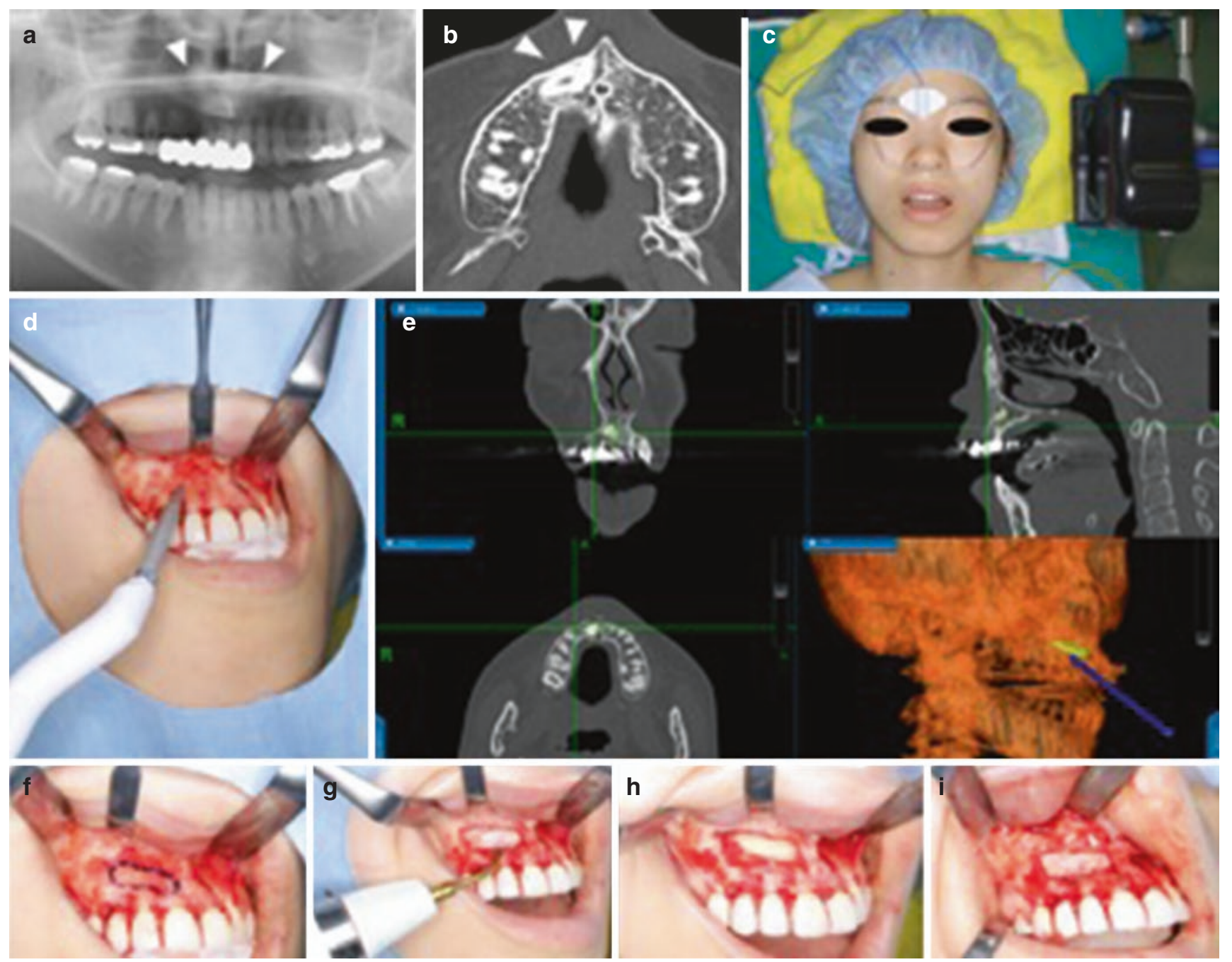

CAssociation of Oral and Maxillofacial Surgeons of India

Fig. 41.16 (a-i) A 24-year-old female with an impacted maxillary canine tooth. Bone lid surgery involves cutting a window into the cortical bone and removing a portion thereof; this portion is subsequently returned to its original position at the end of surgery. Bone lid surgery is a minimally invasive technique designed to avoid secondary large bone defects during osteotomies. However, because jaw bone lesions are completely covered with cortical bone, it can be difficult to accurately determine the position of the lesion from the outside. As a potential solution, we used navigation-assisted bone lid surgery. Using navigation, surgeons could confirm the state of the impacted tooth and surrounding tissue from above the covered bone. We were also able to establish a safe and reliable bone cutting line. In addition, the removed cortical bone was returned to the same (i.e., presurgical) position. Navigation-assisted bone lid surgery for the removal of impacted teeth increases surgical accuracy, minimizes invasion, and allows the bone to be returned to its original position (a, b)-pre operative OPG and CT (c-e)-shows intra operative navigation. (f) outline of canine marked $(\mathbf{g}, \mathbf{h})$ impacted canine being removed (i) the bone lid placed back in position
Wang reported the following advantages of surgical navigation in complicated extractions: (1) localization of teeth for accurate access planning and minimal bone removal; (2) differentiation of impacted teeth from erupting tooth germs; (3) ensured transfer of the preoperative plan to the surgical procedure; and (4) the marking of safety margins while preserving adjacent structures, to avoid complications [40].

\subsubsection{Application for Temporomandibular Joint and Skull Base Surgery}

The TMJ and surrounding anatomy, including the skull base, are extremely complex and require a cautious approach during surgery. Intraoperative navigation can play an important role for surgeries such as the removal of an ankylotic bony mass, tumor resection, and gap arthroplasty [41]. Successful treatment outcomes have been reported by studies for the use 
of navigation assistance in unilateral surgery for the TMJ. Other publications report that navigation is helpful and increases safety in TMJ surgery. A recent publication used navigation to compare prospectively treated groups of patients with recurrent malignant tumors of the infratemporal fossa. Although the results are not independently significant, they yielded a benefit to the navigation cohort. The authors concluded that surgeon confidence and safety during the operations improved, but the navigation system alone did not determine patient outcomes.

Management of tumors at the skull base or of end-stage degenerative TMJ disorders requires thorough knowledge of the regional anatomy and precise 3D planning of the resection margins with attention of vital structures in the immediate vicinity (Fig. 41.17). The location, invasion, and extent of the tumor are key determinants in deciding the surgical approach. In the past, malignant tumors that had infiltrated into the infratemporal fossa or the middle of the skull base were considered inoperable due to the compromised access and inability to achieve predictable tumor control or hemostasis.

The use of surgical navigation for skull base surgery offers the following advantages: (1) ensuring safer and quicker skull base access through a dynamic display of the precise operating site and the extent of bone drilling, thereby significantly reducing intraoperative risk; (2) mapping the anatomical structures and important landmarks such as the foramen ovale and rotundum; and (3) the incorporation of allied imaging modalities, such as 3D CT angiography and MRI, into the intraoperative navigation planning, increasing our understanding of the skull base anatomy and the internal carotid artery region.

As surgery of the skull base is not affected by the shifting brain, use of navigation in this field is more precise than in other neurosurgical procedures. The use of a navigation system for the resection of tumors of the skull base or of TMJ lesions increases surgical predictability while reducing the surgical duration.

\subsubsection{Other Applications}

Another application of intraoperative navigation is in surgery for the management of Eagle's syndrome, which may be due to the elongation of the styloid process of calcification of the stylomandibular ligament [42]. This condition involves a group of symptoms, including throat pain and foreign body sensation on the affected side, reflex otalgia, head and neck pain, and hypersalivation. Surgical treatment was indicated for patients with no symptomatic improvement following conservative treatment.

The surgery may be performed by two different approaches: (1) transcervical and (2) transoral. The cervical approach provides better surgical exposure of the area, but has the major disadvantage of an external incision. The transoral approach is cosmetically favorable, and is more commonly used. However, this approach offers very limited access. This may affect the management of intraoperative complications, including hemorrhage or difficulty in identifying the styloid process. Several methods have been advocated recently to overcome these problems. One report suggests an endoscopically assisted transoral approach for achieving better exposure and visibility of the field. Another method proposes a combination of piezoelectric surgery and surgical navigation for a transcervical approach to remove the styloid. This technique offers a safe and effective method for the treatment of Eagle's syndrome (Fig. 41.18).

\subsection{Recent Advances}

\subsubsection{Navigation Using Intraoperatively Updated Images}

The accuracy of surgical navigation in recent years has been augmented by the induction of intraoperative imaging modalities like the intraoperative CBCT, C-arm, and O-arm systems [43]. These offer intraoperative multiplanar reconstruction capabilities, which enable the improvement of surgical outcomes in demanding surgeries such as surgery of the orbital walls. Furthermore, the popularization of hybrid operating rooms (Fig. 41.19) equipped with both intraoperative imaging and navigation systems has revolutionized surgery of the maxillofacial region. It is now possible to continuously update intraoperative images to determine the best sequence to follow during surgeries (Fig. 41.20). The effectiveness of a navigation system using intraoperative CT images has been already demonstrated in orthopedic surgery and maxillofacial operations, which involve bone movement, such as trauma surgery and orthognathic surgery. Intraoperative CT images have allowed for rapid intraoperative evaluations, which when coupled with surgical navigation, may allow for performing more complicated maxillofacial surgery with increased accuracy. 

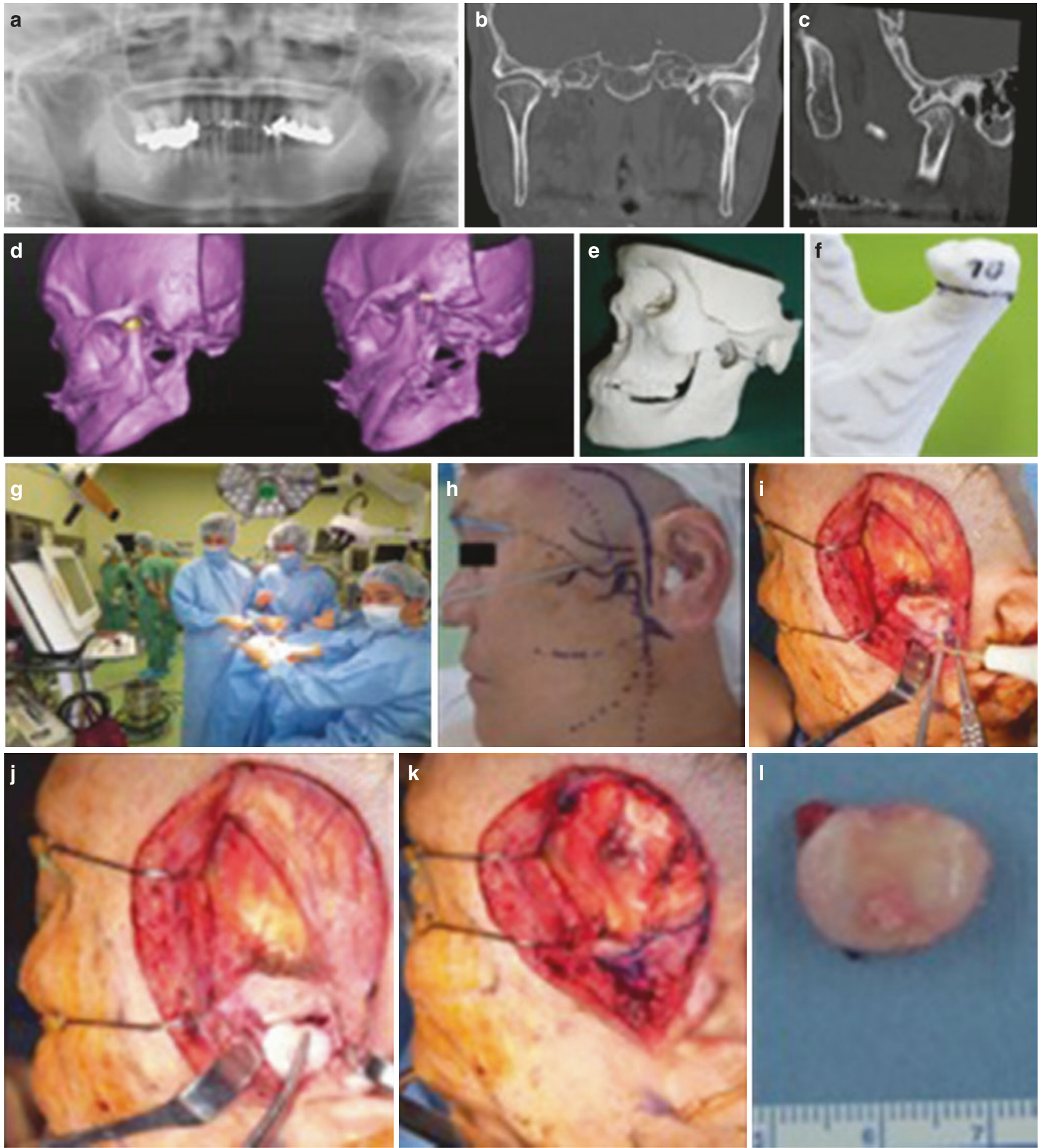

CAssociation of Oral and Maxillofacial Surgeons of India

Fig. 41.17 A 70-year-old female with temporomandibular joint (TMJ) ankylosis. Ankylosis surgery is used for gap arthroplasty and mobilization of the joints. (a-c) However, removal of the bony ankylosis and creation of a gap between the ramus of the mandible and the base of the skull can be difficult because of the size of the ankylosis and the anatomy on the inner aspect of the mandible. Virtual planning is useful in conjunction with surgical navigation to remove the ankylosis. (d) First, a computer simulation based on preoperative CT data was performed for the virtual surgery. (e, f) Once the virtual surgery was completed, templates were constructed using rapid prototyping techniques from the virtual plan and applied at the time of surgery to facilitate the bony cuts. $(\mathbf{g}, \mathbf{h})$ Using the intraoperative navigation system, the surgeon can see the medial aspect of the mandible on the navigation station $\mathrm{CT}$ and protect important structures on the medial side. (i-l) This visualization prevents significant bleeding from the vessels on the medial side of the mandible and prevents penetration into the middle cranial fossa during release of the ankylosis. The temporalis flap was used for prevention of reankylosis 

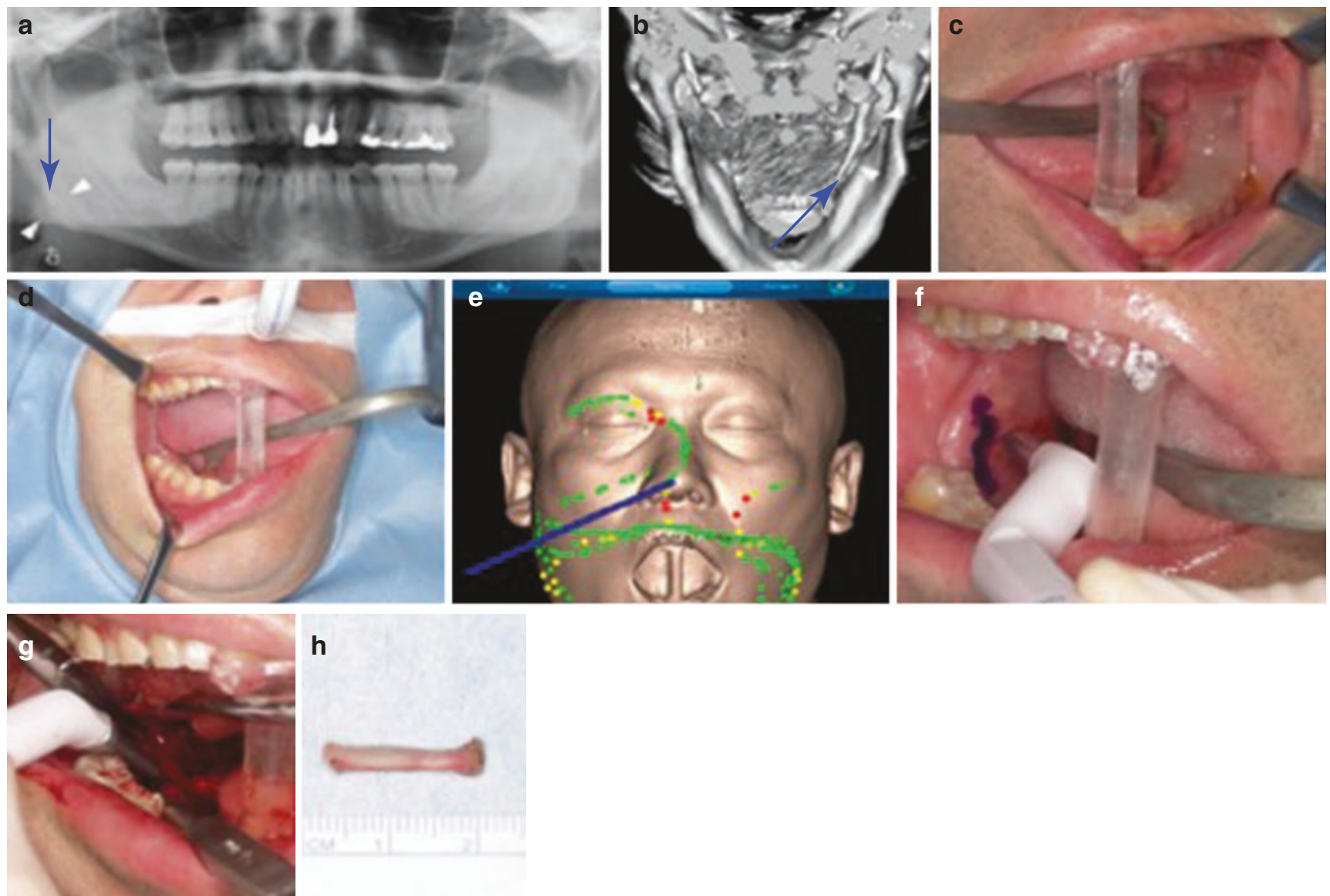

h

CAssociation of Oral and Maxillofacial Surgeons of India

Fig. 41.18 (a, b) A 45-year-old male with elongated styloid process syndrome (Eagle's syndrome). A minimally invasive approach with an intraoperative navigation system was used. (c) Preoperative preparation involved a custom interocclusal splint to produce the mouth opening conditions required during surgery. In this case, since the transoral approach was employed, it was important to reproduce the same mouthopening conditions when obtaining the preoperative images required during surgery, because the position of the styloid process and the blood vessels may change depending on the position of the mandible during the mouth opening. Since it is difficult to implement the locational findings from the preoperative imaging data while performing surgery owing to the mobile nature of the mandible, a custom interocclusal splint for repeated maximum opening in the same mandibular position,

\subsubsection{Wearable Mount Display for Navigation-Assisted Surgery}

Although intraoperative navigation yields helpful information on anatomical features, it is used in conjunction while enabling surgical access, was used. (d) The patient was taken to the operating room, where the custom interocclusal splint was reinserted. (e) To perform patient-to-CT and MRI data registration, the instrumentation navigation probe was used to trace the reference array and soft tissue landmarks of the face. (f) Using the 3D position of the navigation probe, the location of the elongated styloid process was identified. $(\mathbf{g}, \mathbf{h})$ After confirmation of the resection location via the transoral approach, the styloid process was dissected using piezoelectric surgery. Follow-up examination showed an uneventful recovery with no associated complications. (The resection of the styloid process using an intraoperative navigation system and a custom interocclusal splint during a transoral approach, together with a piezoelectric cutting device, is safe and effective for the treatment of Eagle's syndrome)

with a monitor. Generally, the direction of the monitor from the surgeon and operator is different from that the surgical field. Therefore, to see the navigation image, the surgeon and the operator must look up, which is stressful for the operator. It is important to minimize muscle tension and allow the surgeon to perform the operation in a 

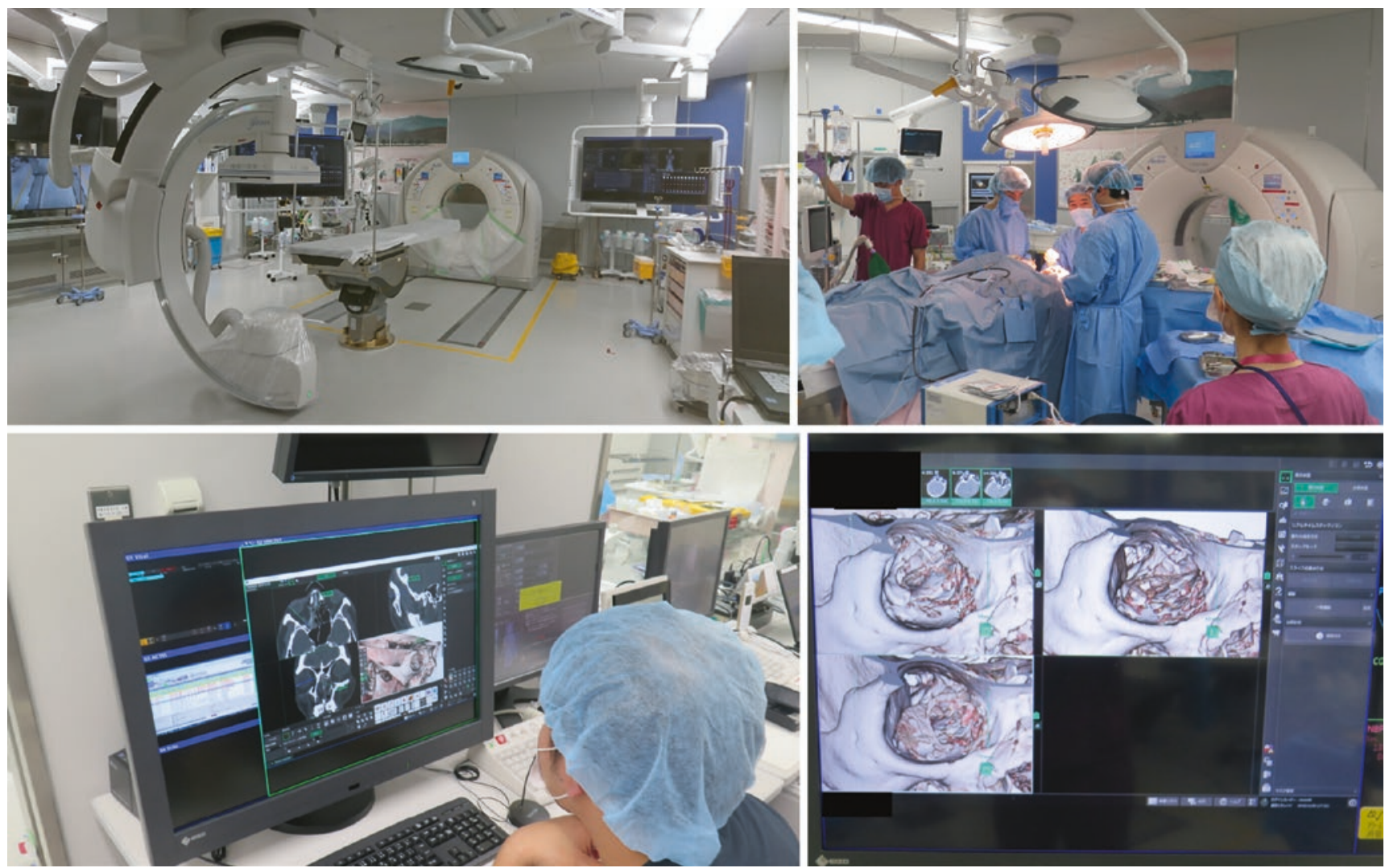

CAssociation of Oral and Maxillofacial Surgeons of India

Fig. 41.19 A hybrid operating room is a surgical theater equipped with advanced medical imaging devices, such as fixed $\mathrm{C}$-arm and angiographic systems. Intraoperative CT imaging is convenient because the
CT is installed in the hybrid operating room. Furthermore, recent advances in technology have made it possible to edit and use images simultaneously with intraoperative CT imaging relaxed position. Moving the head to look at the monitor can result in considerable misalignment of the eye-handtarget axis during task execution, significantly affecting postural comfort and interventional safety. In recent years, head-mounted display monitors have been developed to address this problem. Such wearable displays can provide high-quality images [44]. Using a head-mounted display monitor, surgeons can finish the surgery without moving their head to check the navigation image. The head-mounted device also allows both the surgeon and the assistant to view both the navigation image and the surgical field without interrupting the flow of surgery. This contributes to rapid surgical operation, resulting in minimally invasive surgery. Navigation-assisted surgery with a head-mounted display is a revolutionary technique. In the future, head-mounted displays will be wearable devices that promote the use of navigation (Fig. 41.21).

\subsection{Conclusions and Perspectives}

CAS and navigation offers significant improvements in patient orientation and safety in every facet of maxillofacial surgery. Ranging from precisely planned orthognathic procedures to the removal of foreign bodies requiring 

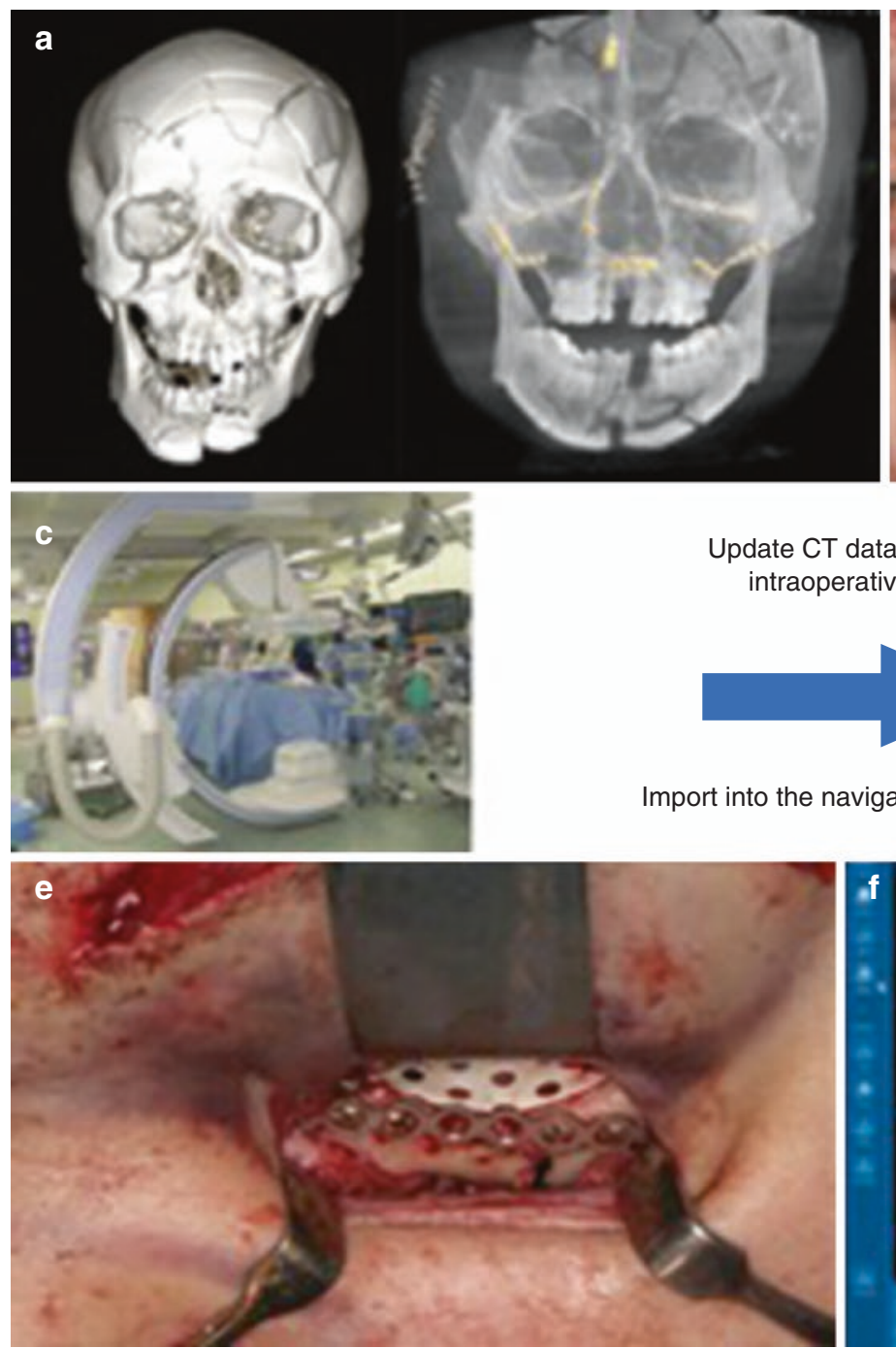

CAssociation of Oral and Maxillofacial Surgeons of India

Fig.41.20 (a) A 19-year-old male with a complex facial fracture and orbital fracture. Navigation surgery is difficult to apply for treating a complex facial fracture with orbital fracture. (b) Since reconstruction of the buttress of the facial bone is performed prior to the treatment of the orbital fracture, preoperative CT data and the actual state of the facial bone are different. Therefore, a navigation system using preoperative

extremely flexible surgical options, and from minimally invasive dental implantology procedures to radical tumor resections of the skull base, they have made their mark improving procedure safety, predictability, and accuracy of surgery while also improving options for intraoperative adaptations. In the future, the application of CAS is expected to further reduce operative risks and surgery
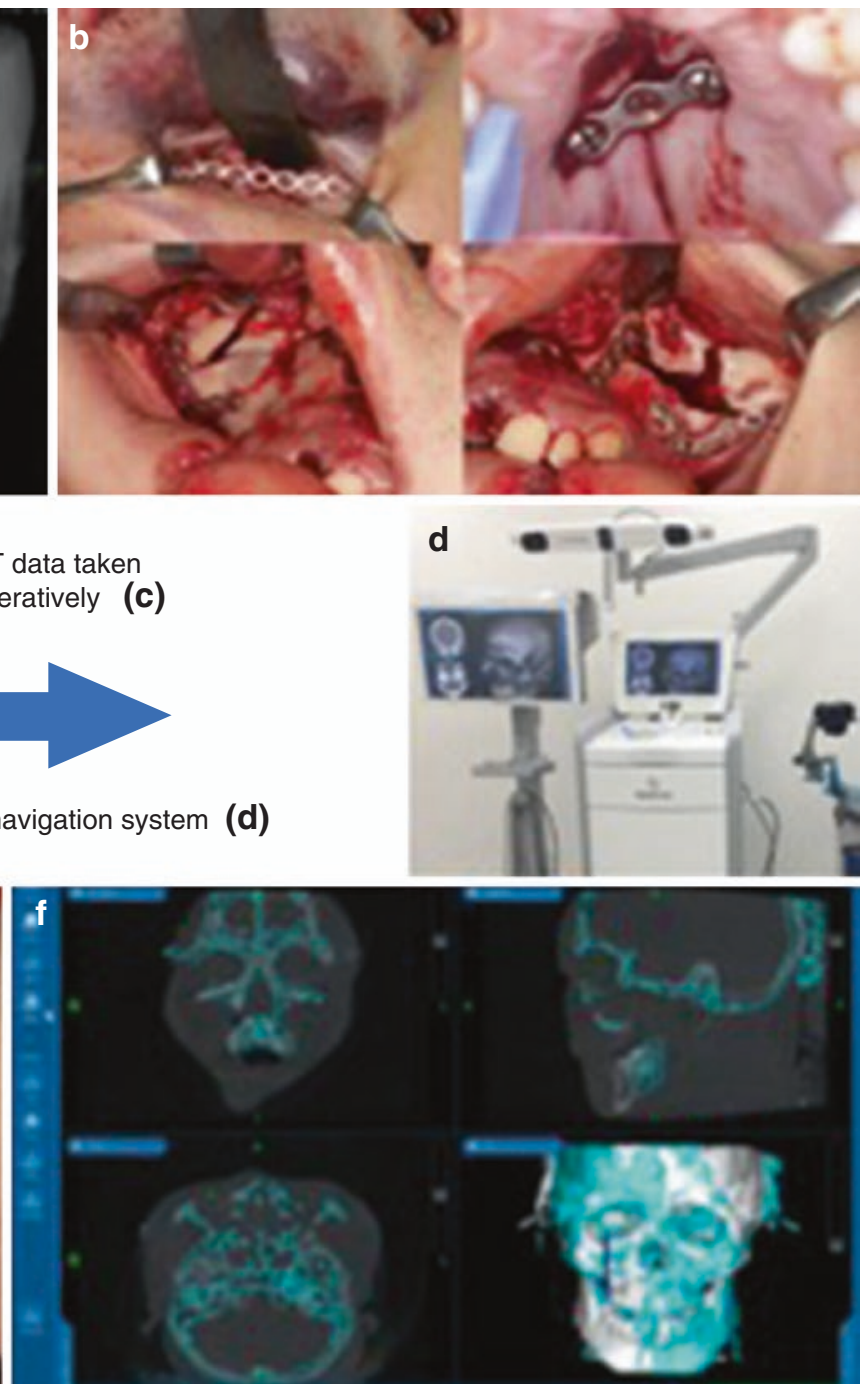

CT data cannot be employed. (c) Using CT data obtained after facial fracture reduction, (d) it is possible to use a navigation system reflecting the updated patient condition. The use of CT in the hybrid room can provide rapid CT data during surgery. (e) The orbital floor defect has been repaired. (f) Post operative CT showing the accurate reduction of fractures

time, accompanied by a considerable decrease in patient stress.

Navigation systems are effective for delicate and accurate oral and maxillofacial surgery and neurosurgery, as well as for otolaryngology and orthopedic surgery. In the future, we expect to develop more convenient and reliable navigation systems using new technologies and devices. 

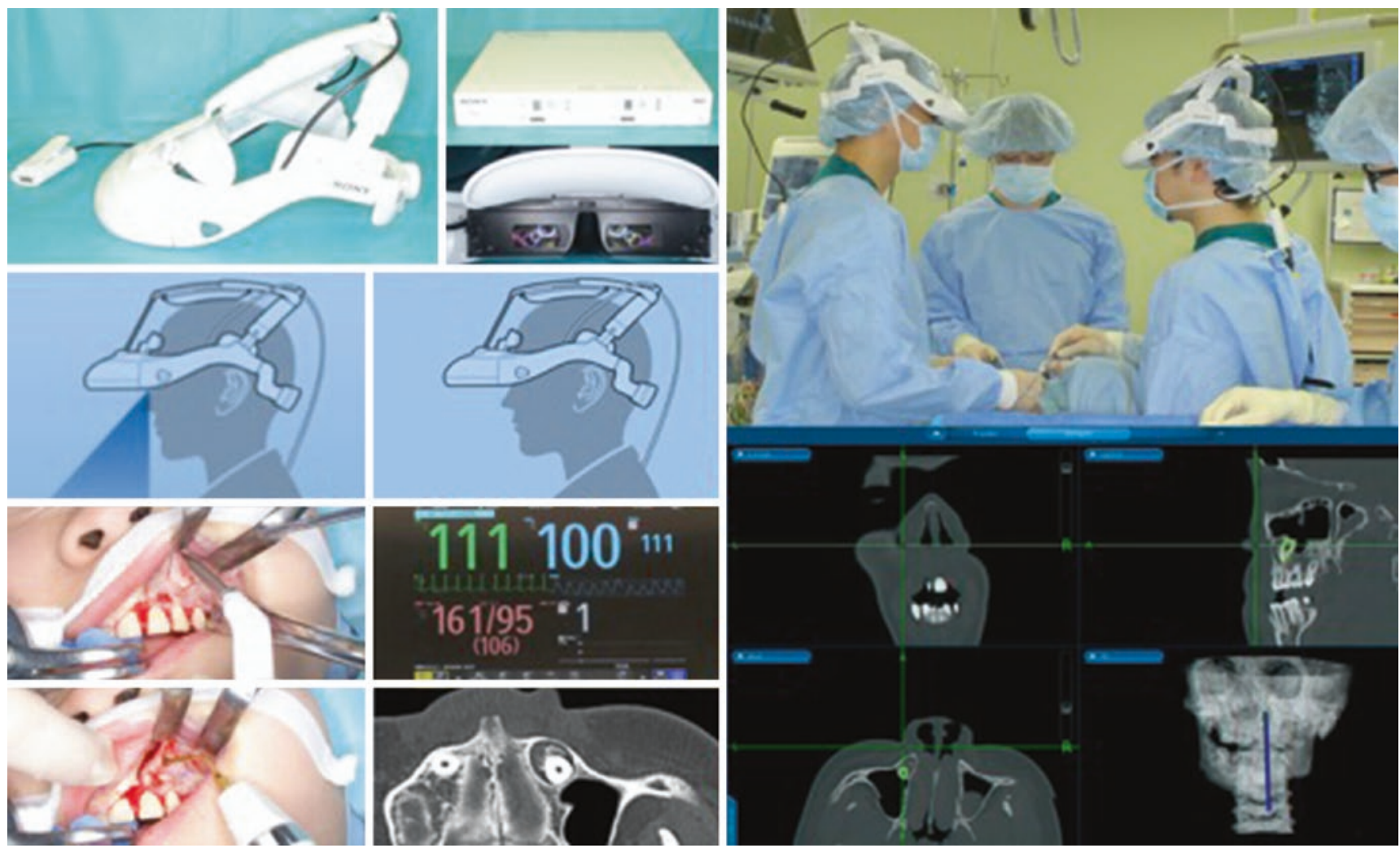

CAssociation of Oral and Maxillofacial Surgeons of India

Fig. 41.21 The head-mounted display can provide high-quality images with wearable technology. The head-mounted display unit was composed of a head-mount image processor unit (HMM-3000MT; Sony Corporation, Tokyo, Japan) and a head-mounted display monitor. Although the navigation system provides helpful information, it is used in conjunction with a monitor. Generally, the direction of the monitor differs from the surgical field. Therefore, to see the navigation image, the surgeon and the operator have to look up, which is stressful for the operator. Using a head-mounted display monitor, we could finish surgery without moving the head position. The surgeon must perform not

\section{References}

1. Sukegawa S, Kanno T, Furuki Y. Application of computer-assisted navigation systems in oral and maxillofacial surgery. Jpn Dent Sci Rev. 2018;54:139-49.

2. Rodby KA, Turin S, Jacobs RJ, Cruz JF, Hassid VJ, Kolokythas A, Antony AK. Advances in oncologic head and neck reconstruction: systematic review and future considerations of virtual surgical planning and computer aided design/computer aided modeling. J Plast Reconstr Aesthet Surg. 2014;67:1171-85.

3. Bell RB. Computer planning and intraoperative navigation in cranio-maxillofacial surgery. Oral Maxillofac Surg Clin N Am. 2010;22:135-56.

4. Kanno T, Sukegawa S, Karino M, Furuki Y. Navigation-assisted orbital trauma reconstruction using a bioactive osteoconductive/bioresorbable u-HA/PLLA system. J Maxillofac Oral Surg. 2019;18:329-38.

5. Li L, Yang J, Chu Y, Wu W, Xue J, Liang P, Chen L. A novel augmented reality navigation system for endoscopic sinus and skull base surgery: a feasibility study. PLoS One. 2016;11:e0146996. only the surgery but also intraoperative systemic management. Multimodality patient information is important in such cases. Multimodality medical information fusion and processing have been developed in recent years. This head-mounted display system allows the integration of preoperative radiological findings with monitoring of navigation images and patient vital signs. Using the head-mounted display system, the surgeon can see multiple images in one view using a split screen, regardless of the head position. This technology facilitates safe surgical management with the navigation system

6. Samarakkody ZM, Abdullah B. The use of image guided navigational tracking systems for endoscopic sinus surgery and skull base surgery: a review. Egypt J Ear Nose Throat Allied Sci. 2016;17:133-7.

7. Hassfeld S, Mühling J, Zöller J. Intraoperative navigation in oral and maxillofacial surgery. Int J Oral Maxillofac Surg. 1995;24:111-9.

8. Marmulla R, Lüth T, Mühling J, Hassfeld S. Markerless laser registration in image-guided oral and maxillofacial surgery. J Oral Maxillofac Surg. 2004;62:845-51.

9. Bettschart C, Kruse A, Matthews F, Zemann W, Obwegeser JA, Grätz KW, Lübbers H-T. Point-to-point registration with mandibulo-maxillary splint in open and closed jaw position. Evaluation of registration accuracy for computer-aided surgery of the mandible. J Cranio-Maxillofac Surg. 2012;40:592-8.

10. Šiniković B, Kramer F-J, Swennen G, Lübbers H-T, Dempf R. Reconstruction of orbital wall defects with calcium phosphate cement: clinical and histological findings in a sheep model. Int J Oral Maxillofac Surg. 2007;36:54-61.

11. Jeon S, Park J, Chien J, Hong J. A hybrid method to improve target registration accuracy in surgical navigation. Minim Invasive Ther Allied Technol. 2015;24:356-63. 
12. Gumprecht HK, Widenka DC, Lumenta CB. Brain lab vector vision neuronavigation system: technology and clinical experiences in 131 cases. Neurosurgery. 1999;44:97-104.

13. Casap N, Wexler A, Eliashar R. Computerized navigation for surgery of the lower jaw: comparison of 2 navigation systems. J Oral Maxillofac Surg. 2008;66:1467-75.

14. Li P, Li Z, Tian W, Tang W. A strategy for removal of foreign body in mandible with navigation system. Int J Oral Maxillofac Surg. 2015;44:885-8.

15. Dong QN, Karino M, Koike T, Ide T, Okuma S, Kaneko I, Osako R, Kanno T. Navigation-assisted isolated medial orbital wall fracture reconstruction using an U-HA/PLLA sheet via a transcaruncular approach. J Investig Surg. 2019:1-9.

16. He D, Li Z, Shi W, Sun Y, Zhu H, Lin M, Shen G, Fan $\mathrm{X}$. Orbitozygomatic fractures with enophthalmos: analysis of 64 cases treated late. J Oral Maxillofac Surg. 2012;70:562-76.

17. Baumann A, Sinko K, Dorner G. Late reconstruction of the orbit with patient-specific implants using computer-aided planning and navigation. J Oral Maxillofac Surg. 2015;73:S101-6.

18. Azarmehr I, Stokbro K, Bell RB, Thygesen T. Surgical navigation: a systematic review of indications, treatments, and outcomes in oral and maxillofacial surgery. J Oral Maxillofac Surg. 2017;75:1987-2005.

19. Schmelzeisen R, Gellrich N-C, Schramm A, Schön R, Otten J-E. Navigation-guided resection of temporomandibular joint ankylosis promotes safety in skull base surgery. J Oral Maxillofac Surg. 2002;60:1275-83.

20. Zrnc TA, Wallner J, Zemann W, Pau M, Gstettner C, Brcic L, Assaf AT, Hassanzadeh H, Feichtinger M, Schwenzer-Zimmerer $\mathrm{K}$. Assessment of tumor margins in head and neck cancer using a 3D-navigation system based on PET/CT image-fusion - a pilot study. J Cranio-Maxillofac Surg. 2018;46:617-23.

21. Sukegawa S, Kanno T, Shibata A, Matsumoto K, SukegawaTakahashi Y, Sakaida K, Furuki Y. Intraoperative navigationassisted accurate bone lid surgery to remove a mandibular lesion: a case report. Oral Maxillofac Surg Cases. 2017;3:15-9.

22. Wei B, Sun G, Hu Q, Tang E. The safety and accuracy of surgical navigation technology in the treatment of lesions involving the skull base. J Craniofac Surg. 2017;28:1431-4.

23. Ricotta F, Cercenelli L, Battaglia S, Bortolani B, Savastio G, Marcelli E, Marchetti C, Tarsitano A. Navigation-guided resection of maxillary tumors: can a new volumetric virtual planning method improve outcomes in terms of control of resection margins? J Cranio-Maxillofac Surg. 2018;46:2240-7.

24. Luna-Ortiz K, Villa-Zepeda O, Carrillo JF, Molina-Frias E, GómezPedraza A. Parapharyngeal space tumor: submandibular approach without mandibulotomy. J Maxillofac Oral Surg. 2018;17:616-24.

25. Mazzoni S, Badiali G, Lancellotti L, Babbi L, Bianchi A, Marchetti C. Simulation-guided navigation. J Craniofac Surg. 2010;21:1698-705.

26. Zinser MJ, Sailer HF, Ritter L, Braumann B, Maegele M, Zöller JE. A paradigm shift in orthognathic surgery? A comparison of navigation, computer-aided designed/computer-aided manufactured splints, and "classic" intermaxillary splints to surgical transfer of virtual orthognathic planning. J Oral Maxillofac Surg. 2013;71:2151.e1-2151.e21.

27. Holly LT, Foley KT. Intraoperative spinal navigation. Spine (Phila. Pa. 1976). 2003;28:S54-61.
28. Block MS, Emery RW. Static or dynamic navigation for implant placement-choosing the method of guidance. J Oral Maxillofac Surg. 2016;74:269-77.

29. Chao AH, Weimer K, Raczkowsky J, Zhang Y, Kunze M, Cody D, Selber JC, Hanasono MM, Skoracki RJ. Pre-programmed robotic osteotomies for fibula free flap mandible reconstruction: a preclinical investigation. Microsurgery. 2016;36:246-9.

30. Sukegawa S, Kawai H, Nakano K, Kanno T, Takabatake K, Nagatsuka H, Furuki Y. Feasible advantage of bioactive/bioresorbable devices made of forged composites of hydroxyapatite particles and poly-L-lactide in alveolar bone augmentation: a preliminary study. Int J Med Sci. 2019;16:311-7.

31. Gaggl A, Schultes G, Kärcher H. Navigational precision of drilling tools preventing damage to the mandibular canal. J CranioMaxillofac Surg. 2001;29:271-5.

32. Stefanelli L, DeGroot B, Lipton D, Mandelaris G. Accuracy of a dynamic dental implant navigation system in a private practice. Int J Oral Maxillofac Implants. 2019;34:205-13.

33. Emery RW, Merritt SA, Lank K, Gibbs JD. Accuracy of dynamic navigation for dental implant placement-model-based evaluation. J Oral Implantol. 2016;42:399-405.

34. Shuker ST, Sadda R. Craniomaxillofacial falling bullet injuries and management. J Oral Maxillofac Surg. 2010;68:1593-601.

35. Bedrock RD, Skigen A, Dolwick MF. Retrieval of a broken needle in the pterygomandibular space. J Am Dent Assoc. 1999;130:685-7.

36. Park S-S, Yang H-J, Lee U-L, Kwon M-S, Kim M-J, Lee J-H, Hwang S-J. The clinical application of the dental mini C-arm for the removal of broken instruments in soft and hard tissue in the oral and maxillofacial area. J Cranio-Maxillofac Surg. 2012;40:572-8.

37. Sukegawa S, Kanno T, Shibata A, Matsumoto K, SukegawaTakahashi Y, Sakaida K, Furuki Y. Use of an intraoperative navigation system for retrieving a broken dental instrument in the mandible: a case report. J Med Case Rep. 2017;11:14.

38. Emery RW, Korj O, Agarwal R. A review of in-office dynamic image navigation for extraction of complex mandibular third molars. J Oral Maxillofac Surg. 2017;75:1591-600.

39. Retana A, Emery RW, Keir V. Removal of impacted supernumerary teeth using a dynamic surgical navigation system: a case report. J Oral Maxillofac Surg. 2019;77:1130-4.

40. Wang H, Yang C-Y, Li Z. Traumatic displacement of teeth into maxillary sinus and the retrieval assisted by computer-assisted navigation. Medicine (Baltimore). 2018;97:e13865.

41. He Y, Huang T, Zhang Y, An J, He L. Application of a computerassisted surgical navigation system in temporomandibular joint ankylosis surgery: a retrospective study. Int J Oral Maxillofac Surg. 2017;46:189-97.

42. Sukegawa S, Kanno T, Yoshimoto A, Matsumoto K, SukegawaTakahashi Y, Masui M, Furuki Y. Use of an intraoperative navigation system and piezoelectric surgery for styloidectomy in a patient with Eagle's syndrome: a case report. J Med Case Rep. 2017;11:322.

43. Wikner J, Riecke B, Gröbe A, Heiland M, Hanken H. Imaging of the midfacial and orbital trauma. Facial Plast Surg. 2014;30:528-36.

44. Matsuo A, Hamada H, Oba H, Shibata K. Virtual reality headmounted display for endoscopically-assisted implant surgery. Br J Oral Maxillofac Surg. 2018;56:636-7.

Open Access This chapter is licensed under the terms of the Creative Commons Attribution 4.0 International License (http://creativecommons. org/licenses/by/4.0/), which permits use, sharing, adaptation, distribution and reproduction in any medium or format, as long as you give appropriate credit to the original author(s) and the source, provide a link to the Creative Commons license and indicate if changes were made.

The images or other third party material in this chapter are included in the chapter's Creative Commons license, unless indicated otherwise in a credit line to the material. If material is not included in the chapter's Creative Commons license and your intended use is not permitted by statutory regulation or exceeds the permitted use, you will need to obtain permission directly from the copyright holder. 